\title{
Multi-gluon helicity amplitudes with one off-shell leg within high energy factorization
}

\section{A. van Hameren, P. Kotko and K. Kutak}

The H. Niewodniczański Institute of Nuclear Physics, Polish Academy of Sciences, Radzikowskiego 152, 31-342 Cracow, Poland

E-mail: Andre.Hameren@ifj.edu.pl, piotr.kotko@ifj.edu.pl, krzysztof .kutak@ifj.edu.pl

ABSTRACT: Basing on the Slavnov-Taylor identities, we derive a new prescription to obtain gauge invariant tree-level scattering amplitudes for the process $g^{*} g \rightarrow N g$ within high energy factorization. Using the helicity method, we check the formalism up to several final state gluons, and we present analytical formulas for the the helicity amplitudes for $N=2$. We also compare the method with Lipatov's effective action approach.

KeYwords: Scattering Amplitudes, QCD

ARXIV EPRINT: 1207.3332 


\section{Contents}

1 Introduction 1

2 Prerequisites and notation 3

2.1 High energy factorization 3

2.2 Color-ordered helicity amplitudes 4

3 Amplitudes with one off-shell leg 5

$\begin{array}{lll}3.1 & \text { Restoration of gauge invariance } & 7\end{array}$

4 Analytical and numerical tests $\quad 11$

4.1 Numerical tests 11

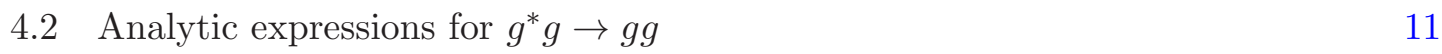

$\begin{array}{lll}4.3 & \text { Comparison with Lipatov's effective action approach } & 13\end{array}$

$\begin{array}{llr}5 & \text { Summary and outlook } & 14\end{array}$

$\begin{array}{ll}\text { A Ghosts and Slavnov-Taylor identities } & 14\end{array}$

B Color-ordered Feynman rules $\quad 16$

$\begin{array}{lll}\text { C Axial gauge and external projectors } & 17\end{array}$

$\begin{array}{ll}\text { D Proof of the 'gauge restoring' amplitude } \mathcal{W} & 19\end{array}$

$\begin{array}{ll}\text { E Singular behaviour of the amplitude } \tilde{\mathcal{A}} & 20\end{array}$

\section{Introduction}

The scientific plans of the Large Hadron Collider (LHC) span from tests of complex dynamics of the Standard Model [1] to searches for physics beyond it [2]. Quantum Chromodynamics (QCD) being a part of the Standard Model is the basic theory which is used to set up the calculational background for the collisions at LHC. Application of perturbative QCD relies on various factorization theorems which allow to decompose a given process into a long-distance part and a short distance hard process. In particular, for inclusive and jet production processes the appropriate long-distance part is realized in terms of various parton distribution functions. Here we will focus on high energy factorization [3] which applies when the energy scale involved in the scattering process is high. The evolution equations of high energy factorization sum up logarithms of energy accompanied by a coupling constant. Depending on the energy range and observable one uses: the BFKL $[4,5]$, 
BK $[6,7]$, CCFM $[8,9]$ or recently proposed new evolution equations accounting for both saturation and processes at large momentum transfers $[10,11]$.

The important ingredients of high energy factorization are unintegrated gluon densities, which depend not only on the longitudinal hadron momentum fraction but on the transversal momentum as well. They have to be convoluted with the hard process which is calculated with the initiating gluons being off-shell. In case of processes with non-gluonic final states, like $q, \bar{q}$ production for instance, the corresponding gauge invariant amplitudes can be calculated by applying so called high energy projectors, that resolve degrees of freedom relevant at high energies. The more general framework given in terms of Lipatov's action [12] provides a prescription to calculate gauge invariant matrix elements in a general case for any partonic process initiated by off-shell gluons [13]. For recent studies and applications, we refer to [14-17].

At present, various automatic numerical tools exist for the calculation of multi-gluon amplitudes for ordinary on-shell processes [18-22]. They operate on the amplitude level and use helicity methods, and thus are very efficient and universal. The present study is a first step aiming at similar methods for off-shell high energy amplitudes. We develop a new prescription for calculating gauge invariant high energy factorizable tree-level amplitudes with one of the gluons being off-shell. Our results turn out to be in agreement with results obtained using Lipatov's action.

Our prescription can be summarized as follows. It is known [23] that the ordinary Feynman graphs contributing to an amplitude in an on-shell calculation are in general not sufficient to obtain gauge invariant results if any of the external legs are off-shell. Thus one usually needs to consider a larger on-shell process and disentangle the required gauge invariant off-shell amplitudes. Our approach is however different: we require the SlavnovTaylor identities to hold for the off-shell connected amplitudes, which in turn induces the necessary contributions which by comparison to [23] can be interpreted as bremsstrahlung graphs introduced to restore gauge invariance. Our construction holds for any number of final state gluons and as an example we give a detailed derivation and discussion for the helicity amplitudes of the process $g^{*} g \rightarrow g g$. These amplitudes are the dominant contribution to forward-central di-jet production [24-26] and are of particular interest for testing parton densities at low longitudinal momentum fraction of the gluons with a special focus on saturation effects (for overview see [27] and for recent jet studies of these effects see $[28])$.

An extensive analysis of tree-level multi-gluon helicity amplitudes in the high-energy limit was done also in ref. [29]. There, the impact factors for $g^{*} g \rightarrow N g, N \leq 3$ were disentangled at the amplitude level, however they do not correspond exactly to the kinematic situation considered in the present work.

The paper is organized as follows. Section 2 is introductory and is mainly devoted to set the notation. In particular, we overview high energy factorization focusing on the kinematic setup and we introduce the framework of color ordered helicity amplitudes which is used later in the paper. In section 3 we identify the problem of gauge non-invariance of high energy factorization amplitudes and repair it using the Slavnov-Taylor identities. In section 4 we describe analytical and numerical tests for gauge invariant helicity amplitudes, 
in particular we list the helicity amplitudes and their square for $g^{*} g \rightarrow g g$. We also cross check our result with those obtained by usage of Lipatov's effective action. In the appendices we collect the color-ordered Feynman rules we needed in our construction of gauge invariant amplitudes as well as some technicalities on the Slavnov-Taylor identities.

\section{Prerequisites and notation}

\subsection{High energy factorization}

Our considerations of the off-shell amplitudes are embedded in the formalism of high energy factorization. Let us thus start with a brief recollection of this topic [3, 30-32].

The basic observation is that the cross section for a hadronic process can be decomposed at high energies into transversal momentum dependent parton densities and the hard partonic cross section with off-shell initial state partons. Thus, the intermediate object that appears is the amplitude for the process

$$
g^{*}\left(k_{1}\right) g^{*}\left(k_{2}\right) \rightarrow X,
$$

where $g^{*}$ denotes an off-shell gluon, and $X$ abbreviates the on-shell final state particles. In the considered high energy limit, the incident momenta are parametrized as

$$
\begin{aligned}
& k_{1}^{\mu} \simeq \xi_{1} n_{a}^{\mu}+k_{1 T}^{\mu}, \\
& k_{2}^{\mu} \simeq \xi_{2} n_{b}^{\mu}+k_{2 T}^{\mu},
\end{aligned}
$$

where $n_{a}, n_{b}$ are light-like momenta corresponding to the two incident hadrons $a$ and $b$. They satisfy $n_{a} \cdot k_{i T}=n_{b} \cdot k_{i T}=0$, and $k_{i}^{2}=k_{i T}^{2}=-\left|\vec{k}_{i T}\right|^{2}$.

In [3], for example, heavy quark production $g^{*}\left(k_{1}\right) g^{*}\left(k_{2}\right) \rightarrow Q \bar{Q}$ is considered. The amplitude is calculated by taking into account the usual tree-level Feynman graphs in the axial gauge with gauge vector $n^{\mu}=\alpha n_{a}^{\mu}+\beta n_{b}^{\mu}$. Then the gluon propagator is given by

$$
D^{\mu \nu}(k)=S(k) d^{\mu \nu}(k, n),
$$

with the scalar part

$$
S(k)=\frac{-i}{k^{2}+i \epsilon}
$$

and the transverse projector

$$
d^{\mu \nu}(k, n)=\eta^{\mu \nu}-\frac{k^{\mu} n^{\nu}+k^{\nu} n^{\mu}}{k \cdot n}+n^{2} \frac{k^{\mu} k^{\nu}}{(k \cdot n)^{2}} .
$$

Above $\eta^{\mu \nu}$ is the metric tensor and we have suppressed the color indices in $D^{\mu \nu}$. The off-shell legs include propagators, and are contracted with eikonal couplings defined as

$$
\begin{aligned}
\mathfrak{e}_{1}^{\mu} & =\left|\vec{k}_{1 T}\right| n_{a}^{\mu}, \\
\mathfrak{e}_{2}^{\mu} & =\left|\vec{k}_{2 T}\right| n_{b}^{\mu} .
\end{aligned}
$$


It is easy to check, that effectively an amputated amplitude is contracted with the following vector

$$
-i \mathfrak{e}_{1}^{\mu} D_{\mu \nu}\left(k_{1}\right)=\frac{k_{T 1}^{\mu}}{\xi_{1}\left|\vec{k}_{1 T}\right|}
$$

and similarly for $k_{2}$, which then is shown to lead to the correct result in the collinear limit. Furthermore, it is shown that the result is independent of the choice of gauge-vector, proving gauge invariance.

The above might be taken as a prescription to calculate amplitudes for arbitrary processes, but it will in general not lead to gauge-invariant results. To achieve the latter, additional contributions are needed. They are usually obtained by considering a larger, fully on-shell, process from which an amplitude for the off-shell process is extracted. The situation is somewhat simpler when one of the gluons, say $k_{2}$, reaches collinear limit, i.e. when $k_{2}^{2} \rightarrow 0$. In the following sections, we shall investigate such amplitudes for the process

$$
g^{*}\left(k_{1}\right) g\left(k_{2}\right) \rightarrow g\left(k_{3}\right) \ldots g\left(k_{N}\right)
$$

for an arbitrary number of final state gluons.

\subsection{Color-ordered helicity amplitudes}

Let us denote our purely gluonic tree-level amplitude with $N$ external gluons (one offshell and $N-1$ on-shell) as $\mathcal{M}\left(\mathfrak{e}_{1}, \varepsilon_{2}, \ldots, \varepsilon_{N}\right)$. For the precise definition see section 3 . The polarization vectors of the on-shell gluons are denoted as $\varepsilon_{i}$. Whenever necessary, we shall indicate the polarization state explicitly using $\varepsilon_{i}^{(\lambda)}$ with $\lambda= \pm$. Following e.g. the Kleiss-Stirling construction [33], the polarization vectors can be defined in terms of the gluon momentum $k_{i}$ and an auxiliary light-like momentum $q_{i}$ satisfying $k_{i} \cdot q_{i} \neq 0$. Thus, whenever needed we use the notation $\varepsilon_{i}^{(\lambda)}\left(q_{i}\right)$ to indicate explicitly what reference vector is used. The polarization vectors satisfy several conditions, of which we would like to highlight two, namely

$$
\begin{gathered}
\varepsilon_{i}^{(\lambda)}\left(q_{i}\right) \cdot k_{i}=0, \\
\varepsilon_{i}^{(\lambda)}\left(q_{i}\right) \cdot q_{i}=0 .
\end{gathered}
$$

When one changes the reference momentum, the corresponding polarization vector transforms as

$$
\varepsilon_{i}^{\mu}\left(q_{i}^{\prime}\right)=\varepsilon_{i}^{\mu}\left(q_{i}\right)+\beta_{i}\left(q_{i}^{\prime}, q_{i}\right) k_{i}^{\mu},
$$

where the function $\beta_{i}$ depends on the precise representation for the polarization vectors. It is important to note, that as long as the amplitude $\mathcal{M}$ satisfies the Ward identity

$$
\mathcal{M}\left(\mathfrak{e}_{1}, \varepsilon_{2}, \ldots, \varepsilon_{i-1}, k_{i}, \varepsilon_{i+1}, \ldots, \varepsilon_{N}\right)=0, \quad i=2, \ldots, N
$$

the reference momenta can be freely changed, which has proved to be very useful for obtaining compact expressions for multi-gluon amplitudes (see e.g. [34] for a review). In explicit calculations we use the spinor representation for the polarization vectors

$$
\varepsilon_{i}^{\mu \pm}\left(q_{i}\right)= \pm \frac{\left\langle q_{i}^{\mp}\left|\gamma^{\mu}\right| k_{i}^{\mp}\right\rangle}{\sqrt{2}\left\langle q_{i}^{\mp} \mid k_{i}^{ \pm}\right\rangle},
$$


where

$$
\begin{aligned}
\left\langle k_{i}^{-} \mid k_{j}^{+}\right\rangle & =\bar{u}^{-}\left(k_{i}\right) u^{+}\left(k_{j}\right) \equiv\langle i j\rangle, \\
\left\langle k_{i}^{+} \mid k_{j}^{-}\right\rangle & =\bar{u}^{+}\left(k_{i}\right) u^{-}\left(k_{j}\right) \equiv[i j]
\end{aligned}
$$

and the positive-energy spinors with definite helicities are defined as $u^{ \pm}\left(k_{i}\right)=$ $\frac{1}{2}\left(1 \pm \gamma_{5}\right) u\left(k_{i}\right)$. The spinor products can be explicitly calculated in the light-cone basis as

$$
\begin{aligned}
& \langle i j\rangle=k_{\perp i} \sqrt{\frac{k_{j}^{+}}{k_{i}^{+}}}-k_{\perp j} \sqrt{\frac{k_{i}^{+}}{k_{j}^{+}}}, \\
& {[i j]=-k_{\perp i}^{*} \sqrt{\frac{k_{j}^{+}}{k_{i}^{+}}}+k_{\perp j}^{*} \sqrt{\frac{k_{i}^{+}}{k_{j}^{+}}},}
\end{aligned}
$$

where $k^{ \pm}=k^{0} \pm k^{3}$ and $k_{\perp}=k^{1}+i k^{2}$. We also use the following compact notation for spinor products with some $\not$ matrix insertion

$$
\begin{aligned}
& \left\langle q^{-}|\not| k^{-}\right\rangle \equiv\langle q|\not| k], \\
& \left\langle q^{+}|\not| \mid k^{+}\right\rangle \equiv[q|\not| k|k\rangle \text {. }
\end{aligned}
$$

Another important ingredient in this respect is the use of color-ordered amplitudes [35]. They are defined via

$$
\mathcal{M}\left(\mathfrak{e}_{1}, \varepsilon_{2}, \ldots, \varepsilon_{N}\right)=\sum_{\begin{array}{c}
\text { non-cyclic } \\
\text { permutations }
\end{array}} \operatorname{Tr}\left(t^{a_{1}} \ldots t^{a_{N}}\right) \mathcal{A}\left(\mathfrak{e}_{1}, \varepsilon_{2}, \ldots, \varepsilon_{N}\right)
$$

where the summation is over all non-cyclic permutations of the color indices $\left\{a_{1}, \ldots, a_{N}\right\}$ corresponding to the external gluons. The matrices $t^{a}$ are the generators of color $\mathrm{SU}\left(N_{c}\right)$ group normalized as $\operatorname{Tr}\left(t^{a} t^{b}\right)=\delta^{a b}$. Note, that the ordering of the arguments in $\mathcal{A}$ does matter and corresponds to the order of color matrices under the trace. There are several important properties of color-ordered amplitudes. Here we want to mention explicitly two of them. First, gauge invariance of $\mathcal{M}$ implies gauge invariance of $\mathcal{A}$. Second, the full amplitude squared is given by

$$
\sum_{\text {colors }}\left|\mathcal{M}\left(\mathfrak{e}_{1}, \varepsilon_{2}, \ldots, \varepsilon_{N}\right)\right|^{2}=N_{c}^{N-2}\left(N_{c}^{2}-1\right) \sum_{\begin{array}{c}
\text { non-cyclic } \\
\text { permutations }
\end{array}}\left|\mathcal{A}\left(\mathfrak{e}_{1}, \varepsilon_{2}, \ldots, \varepsilon_{N}\right)\right|^{2}+\mathcal{O}\left(N_{c}^{N-2}\right),
$$

where the contribution $\mathcal{O}\left(N_{c}^{N-2}\right)$ vanishes for $N \leq 5$.

\section{Amplitudes with one off-shell leg}

The subject of our investigations are gluonic amplitudes with one off-shell leg, eq. (2.10). More precisely, they are obtained from the corresponding momentum space Green function 


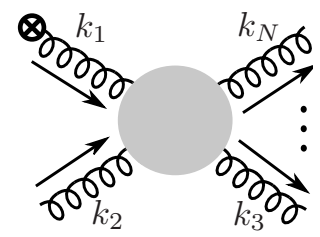

Figure 1. The color-ordered amplitude $\mathcal{A}\left(\mathfrak{e}_{1}, \varepsilon_{2}, \ldots, \varepsilon_{N}\right)$ defined in the main text. The special external vertex corresponds to eikonal coupling $\mathfrak{e}_{1}^{\mu}=\left|\vec{k}_{T}\right| n_{a}^{\mu}$, while all the other external lines are amputated and contracted with the corresponding polarization vectors. The arrows show the choice of the momenta signs, while the vertical dots stand for the remaining external gluons.

by means of the following reduction formula

$$
\begin{aligned}
\mathcal{M}\left(\mathfrak{e}_{1}, \varepsilon_{2}, \ldots, \varepsilon_{N}\right)=\lim _{k_{1} \cdot n_{a} \rightarrow 0} \lim _{k_{2}^{2}, \ldots, k_{N}^{2} \rightarrow 0} \mathfrak{e}_{1}^{\mu_{1}} \varepsilon_{2}^{\mu_{2}} \ldots \varepsilon_{N}^{\mu_{N}} \\
S^{-1}\left(k_{2}\right) \ldots S^{-1}\left(k_{N}\right) \tilde{G}_{\mu_{1} \ldots \mu_{N}}\left(k_{1}, \ldots, k_{N}\right),
\end{aligned}
$$

where the scalar propagators are defined in eq. (2.5) and $\tilde{G}$ is momentum space Green function defined as usual, by Fourier transforming the vacuum matrix element of a time ordered product of gluon fields. Note, that the first limit is a formal way of implementing the kinematic condition (2.2). The amplitude defined in eq. (3.1) is incidentally not gauge invariant, see the discussion below.

Let us remark, that for ordinary, completely on-shell amplitudes the common practice is to amputate the external gluon legs together with potential projectors (2.6), if one uses axial gauge for $\tilde{G}$. In other words, in such case we can choose different gauges for the internal (off-shell) and the external (on-shell) lines and the scattering amplitude does not depend on this choice. It is of course also possible to do so at intermediate stages of a calculation when dealing with gauge-dependent quantities, however one has to be consistent, as different gauge-dependent pieces are to eventually compose a gauge invariant object.

In this spirit, in what follows we work in the axial gauge with $n_{a}$ being the gauge vector for internal off-shell lines (including line $k_{1}$ ) while the external on-shell gluons are reduced in the Feynman gauge, unless otherwise specified. That is, any vertex adjacent to an external on-shell gluon is contracted with the corresponding polarization vector directly.

In what follows, we consider a color-ordered amplitude for some specific color ordering, say $\left(a_{1}, a_{2}, \ldots, a_{N}\right)$, where $a_{i}$ is the color quantum number of the gluon with momentum $k_{i}$. As follows from (3.1), the off-shell leg $k_{1}$ contains the full propagator and is contracted with the eikonal vertex $\mathfrak{e}_{1}$ defined in eq. (2.7), which we graphically denote as a circle with a cross (figure 1). According to section 2.2, the amplitude under consideration is denoted as $\mathcal{A}\left(\mathfrak{e}_{1}, \varepsilon_{2}, \ldots, \varepsilon_{N}\right)$. For simplicity, we choose

$$
k_{1}^{\mu}=n_{a}^{\mu}+k_{T}^{\mu}
$$

as the momentum of the off-shell gluon (i.e. we absorb $\xi$ into $n_{a}$ in eq. (2.2)). 
Because of the off-shell external leg, taking into account the usual tree-level Feynman graphs and using the usual Feynman rules will not lead to a gauge invariant amplitude. In particular, it will not satisfy the usual Ward identities, i.e.

$$
\mathcal{A}\left(\mathfrak{e}_{1}, \varepsilon_{2}, \ldots, \varepsilon_{i-1}, k_{i}, \varepsilon_{i+1}, \ldots, \varepsilon_{N}\right) \neq 0, \quad i=2, \ldots, N
$$

which are indispensable for the calculation of helicity amplitudes. Indeed, it is not the Ward identity of the above form that follows from the local gauge invariance. Rather, one should consider the non-abelian Slavnov-Taylor (S-T) identites, which relate the amplitude $\mathcal{A}$ with nonphysical polarization (i.e. insertion of $k_{i}$ ) and the corresponding diagrams with ghosts. In the next section, we shall wander this path to construct a new amplitude

$$
\tilde{\mathcal{A}}\left(\mathfrak{e}_{1}, \varepsilon_{2}, \ldots, \varepsilon_{N}\right)=\mathcal{A}\left(\mathfrak{e}_{1}, \varepsilon_{2}, \ldots, \varepsilon_{N}\right)+\mathcal{W}\left(\mathfrak{e}_{1}, \varepsilon_{2}, \ldots, \varepsilon_{N}\right),
$$

such that

$$
\tilde{\mathcal{A}}\left(\mathfrak{e}_{1}, \varepsilon_{2}, \ldots, \varepsilon_{i-1}, k_{i}, \varepsilon_{i+1}, \ldots, \varepsilon_{N}\right)=0, \quad i=2, \ldots, N .
$$

Since we provide a prescription to calculate $\tilde{\mathcal{A}}\left(\mathfrak{e}_{1}, \varepsilon_{2}, \ldots, \varepsilon_{N}\right)$ in a specific gauge, the fact that it satisfies eq. (3.5) is an indication that it is the correct gauge invariant result. Moreover, we show in appendix E that (3.4) posseses correct collinear and soft behaviour in the corresponding limits.

\subsection{Restoration of gauge invariance}

In what follows, we skip the first argument (corresponding to the off-shell leg) in the colorordered amplitude $\mathcal{A}$, i.e. we write $\mathcal{A}\left(\mathfrak{e}_{1}, \varepsilon_{2}, \ldots, \varepsilon_{N}\right) \equiv \mathcal{A}\left(\varepsilon_{2}, \ldots, \varepsilon_{N}\right)$ for compactness. In the gauge we specified above, our solution for the 'gauge restoring' amplitude $\mathcal{W}$ reads

$$
\mathcal{W}\left(\varepsilon_{2}, \ldots, \varepsilon_{N}\right)=\left(-\frac{g}{\sqrt{2}}\right)^{N-2} \frac{-\left|\vec{k}_{T}\right| \varepsilon_{2} \cdot n_{a} \ldots \varepsilon_{N} \cdot n_{a}}{k_{2} \cdot n_{a}\left(k_{2}-k_{3}\right) \cdot n_{a} \ldots\left(k_{2}-\ldots-k_{N-1}\right) \cdot n_{a}} .
$$

The above formula can be interpreted as a straight Wilson line along $n_{a}$ with the external gluons attached to it, however such an interpretation is beyond the scope of this paper and shall be analyzed elsewhere.

As already mentioned, according to the S-T identities, the insertion of $k_{i}$ generates additional gauge terms on the r.h.s. of (3.3). We shall see that by controlling those terms one can indeed reconstruct $\mathcal{W}$. The $\mathrm{S}-\mathrm{T}$ identities in different gauges together with some less common issues are recollected in appendix $\mathrm{A}$.

Let us consider first the (color-ordered) momentum space Green function, from which our amplitude is obtained according to the reduction procedure (3.1). Since we have chosen the Feynman gauge for the external on-shell lines, the relevant S-T identity involves the space-time derivative of the fields and in turn translates into a momentum insertion as in (3.3), c.f. (A.9).

The S-T identities for the Green functions are most conveniently given in graphical form. We show an example in figure $2 \mathrm{~A}$ for the contraction with $k_{2}$. It generates a set of diagrams involving ghosts and picks out a ghost-gluon vertex (see (A.10) in appendix A) 
A)

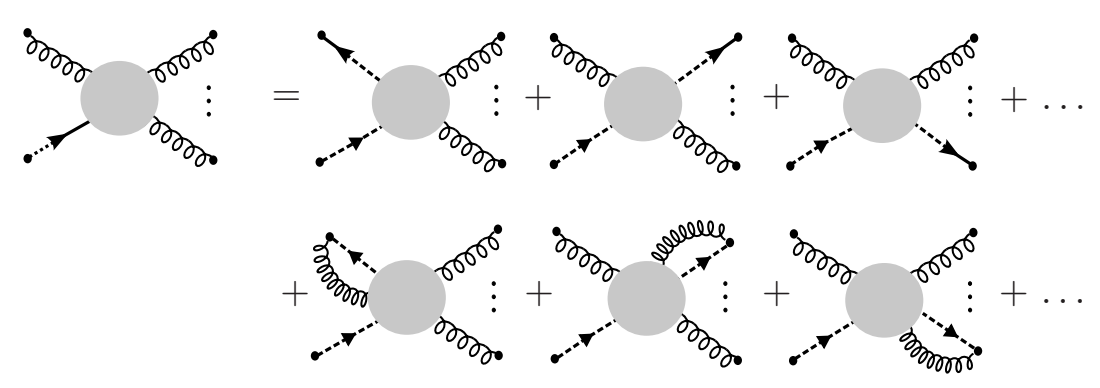

B)
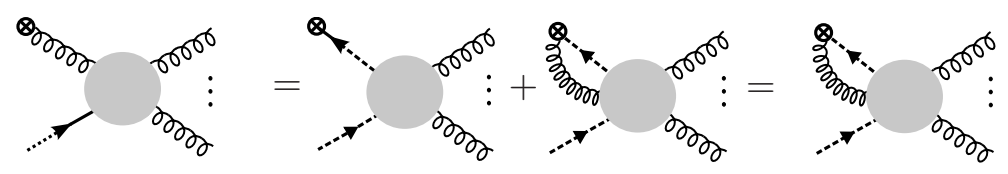

Figure 2. A) The Slavnov-Taylor identity for the Green function (with external propagators) contracted with $k_{2}$. The contraction is graphically denoted by the arrow transforming to a solid line (see appendix B). The dashed lines with arrows are standard notation for ghosts. Horizontal dots denote analogous diagrams for the rest of external legs. B) Application of the identity for the amplitude for a process with $N-1$ on-shell gluons and one off-shell leg contracted with an eikonal vertex. Most of the gauge diagrams vanish as described in the main text, but not all due to the off-shellness of $k_{1}$.

without an external propagator. The ghosts propagating to the shaded blob, which is assumed to be calculated in the axial gauge, may need some explanation. Let us note that the ghosts can be introduced also in the axial gauge but they decouple for ordinary processes, see e.g. [36] and appendix A for additional explanations. The ghost-gluon coupling is proportional to $n_{a}^{\mu}$ and is transverse to the gluon propagator in the axial gauge $n_{a}^{\mu} d_{\mu \nu}\left(k, n_{a}\right)=0$, thus any internal gluon cannot be coupled to a ghost. In our case, however, a ghost can couple to external on-shell gluons, which are taken in the Feynman gauge and for which the projector $d_{\mu \nu}$ is absent.

When going from the Green function to the amplitude $\mathcal{A}$ most of the additional gauge diagrams vanish (figure $2 \mathrm{~B}$ ), either due to the transversality condition (2.11), or because the residue at the physical pole is zero (due to the lack of the external propagator, c.f. the reduction formula (3.1)). However, since $k_{1}$ is off-shell eventually one term survives, namely the one with the ghost-gluon vertex with the amputated external line (the second term of BRST transformation vertex (A.10)) contracted with the eikonal coupling. Note, that the first term in the middle in figure $2 \mathrm{~B}$ vanishes due to the relation $k_{1} \cdot n_{a}=0$.

In order to illustrate the above statements, let us look at the specific realization of the gauge diagrams from figure $2 \mathrm{~B}$ for the simplest process $g^{*} g \rightarrow g g$. We display the diagrams in figure 3 for the color-ordered amplitude. Since we work in the axial gauge for the internal lines, no gluon line can attach to a ghost unless it is an external line. There is only one possible diagram with a triple-gluon coupling in which it is connected to the eikonal vertex (right diagram in figure 3). But this diagram is also zero, since we have chosen $n_{a}$ as the gauge vector. 

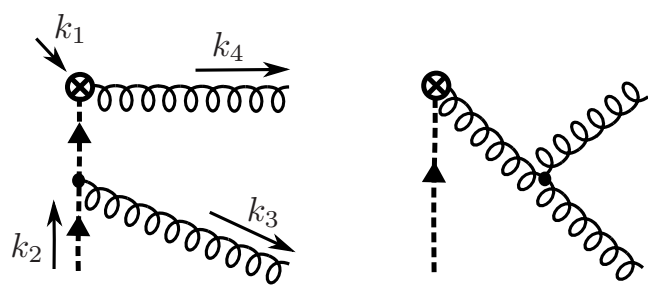

Figure 3. The diagrams contributing to the Slavnov-Taylor identity for the color-ordered amplitude $\mathcal{A}\left(\mathfrak{e}_{1}, \varepsilon_{2}, \varepsilon_{3}, \varepsilon_{4}\right)$, corresponding to the the process $g^{*} g \rightarrow g g$. We show the momentum flow in the leftmost diagram only. The right diagram is zero if the axial gauge with $n_{a}$ gauge vector is chosen.

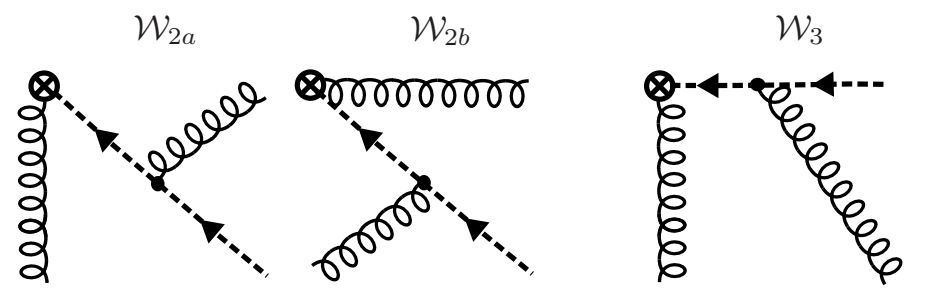

Figure 4. The color-ordered gauge diagrams for the contractions of the amplitude $\mathcal{A}\left(\varepsilon_{2}, \varepsilon_{3}, \varepsilon_{4}\right)$ with the external momenta; here $\mathcal{W}_{2 a}$ and $\mathcal{W}_{2 b}$ correspond to $\mathcal{A}\left(\varepsilon_{2}, k_{3}, \varepsilon_{4}\right)$, while $\mathcal{W}_{3}$ corresponds to $\mathcal{A}\left(\varepsilon_{2}, \varepsilon_{3}, k_{4}\right)$. The momentum flow is the same as in figure 3 .

The remaining gauge diagrams can easily be calculated and one can explicitly check the S-T identities (we checked it also for a general axial gauge with gauge vector $n \neq n_{a}$ ). For instance, for the left diagram from figure 3 we obtain using the Feynman rules collected in appendix B

$$
\frac{g^{2}}{2}\left|\vec{k}_{T}\right| \frac{n_{a} \cdot \varepsilon_{3} n_{a} \cdot \varepsilon_{4}}{\left(k_{2}-k_{3}\right) \cdot n_{a}} \equiv \mathcal{W}_{1}\left(k_{2}, \varepsilon_{3}, \varepsilon_{4}\right) .
$$

The arguments of $\mathcal{W}_{1}$ are chosen in this way for further convenience and show what leg is contracted with the momentum in the corresponding S-T relation.

We can derive analogous identities involving contractions for different external legs. The corresponding gauge diagrams are depicted in figure 4. Using analogous naming convention as in eq. (3.7) they read

$$
\begin{aligned}
& \mathcal{W}_{2 a}\left(\varepsilon_{2}, k_{3}, \varepsilon_{4}\right)=\frac{g^{2}}{2}\left|\vec{k}_{T}\right| \frac{n_{a} \cdot \varepsilon_{2} n_{a} \cdot \varepsilon_{4}}{\left(k_{1}+k_{2}\right) \cdot n_{a}}, \\
& \mathcal{W}_{2 b}\left(\varepsilon_{2}, k_{3}, \varepsilon_{4}\right)=\frac{g^{2}}{2}\left|\vec{k}_{T}\right| \frac{n_{a} \cdot \varepsilon_{2} n_{a} \cdot \varepsilon_{4}}{\left(k_{1}-k_{4}\right) \cdot n_{a}}, \\
& \mathcal{W}_{3}\left(\varepsilon_{2}, \varepsilon_{3}, k_{4}\right)=-\frac{g^{2}}{2}\left|\vec{k}_{T}\right| \frac{n_{a} \cdot \varepsilon_{2} n_{a} \cdot \varepsilon_{3}}{\left(k_{1}+k_{2}\right) \cdot n_{a}} .
\end{aligned}
$$

An immediate observation is that the gauge diagrams are proportional to $\left|\vec{k}_{T}\right|$, thus they vanish when all the particles become on-shell; consequently the l.h.s. of (3.3) equals to zero in that limit.

The 'gauge restoring' amplitude $\mathcal{W}$ in (3.4) can be obtained using the above results. First, we define $\mathcal{W}_{x}\left(\varepsilon_{2}, \varepsilon_{3}, \varepsilon_{4}\right)$ (note the arguments) for $x=1,2 a, 2 b, 3$ by multiplying the 
external ghost lines with the longitudinal projections of the corresponding polarization vectors. More precisely, we multiply the ghost leg $i$ with $\varepsilon_{i} \cdot n_{a} / k_{i} \cdot n_{a}$. Hence we have

$$
\begin{aligned}
\mathcal{W}_{1}\left(\varepsilon_{2}, \varepsilon_{3}, \varepsilon_{4}\right) & =-\mathcal{W}_{3}\left(\varepsilon_{2}, \varepsilon_{3}, \varepsilon_{4}\right)=\frac{g^{2}}{2}\left|\vec{k}_{T}\right| \frac{n_{a} \cdot \varepsilon_{2} n_{a} \cdot \varepsilon_{3} n_{a} \cdot \varepsilon_{4}}{k_{2} \cdot n_{a} k_{4} \cdot n_{a}} \\
\mathcal{W}_{2 a}\left(\varepsilon_{2}, \varepsilon_{3}, \varepsilon_{4}\right) & =\frac{g^{2}}{2}\left|\vec{k}_{T}\right| \frac{n_{a} \cdot \varepsilon_{2} n_{a} \cdot \varepsilon_{3} n_{a} \cdot \varepsilon_{4}}{k_{2} \cdot n_{a} k_{3} \cdot n_{a}} \\
\mathcal{W}_{2 b}\left(\varepsilon_{2}, \varepsilon_{3}, \varepsilon_{4}\right) & =-\frac{g^{2}}{2}\left|\vec{k}_{T}\right| \frac{n_{a} \cdot \varepsilon_{2} n_{a} \cdot \varepsilon_{3} n_{a} \cdot \varepsilon_{4}}{k_{3} \cdot n_{a} k_{4} \cdot n_{a}}
\end{aligned}
$$

Then, we define

$$
\mathcal{W}\left(\varepsilon_{2}, \varepsilon_{3}, \varepsilon_{4}\right)=\mathcal{W}_{1}\left(\varepsilon_{2}, \varepsilon_{3}, \varepsilon_{4}\right)+\mathcal{W}_{2 a}\left(\varepsilon_{2}, \varepsilon_{3}, \varepsilon_{4}\right)+\mathcal{W}_{2 b}\left(\varepsilon_{2}, \varepsilon_{3}, \varepsilon_{4}\right)+\mathcal{W}_{3}\left(\varepsilon_{2}, \varepsilon_{3}, \varepsilon_{4}\right)
$$

Adding the diagrams, we recover the result anticipated in eq. (3.6) for $N=4$

$$
\mathcal{W}\left(\varepsilon_{2}, \varepsilon_{3}, \varepsilon_{4}\right)=-\frac{g^{2}}{2}\left|\vec{k}_{T}\right| \frac{n_{a} \cdot \varepsilon_{2} n_{a} \cdot \varepsilon_{3} n_{a} \cdot \varepsilon_{4}}{k_{2} \cdot n_{a} k_{4} \cdot n_{a}} .
$$

It is straightforward to check that indeed (3.5) is satisfied with this choice of $\mathcal{W}$. It is a general property of $\mathcal{W}$ in our choice of gauge, that the sum of the diagrams with ghosts (and further replaced by longitudinal gluon polarization) collapses to single terms. The complete proof of eq. (3.6) for any number of gluons is relegated to appendix D.

We want to close this section with the following remark. Note that if we choose the reference momentum for any of the polarization vectors to be $n_{a}$, the 'gauge restoring' amplitude is zero

$$
\mathcal{W}\left(\varepsilon_{2}, \ldots, \varepsilon_{i-1}, \varepsilon_{i}\left(n_{a}\right), \varepsilon_{i+1}, \ldots, \varepsilon_{N}\right)=0
$$

for any leg $i$. It follows from the property of polarization vectors (2.12). Let us now note, that we can always project a polarization vector with any reference momentum $q_{i}$ to the one with $n_{a}$ using

$$
\varepsilon_{i}^{\mu}\left(q_{i}\right) d_{\mu}^{\nu}\left(k_{i}, n_{a}\right)=\varepsilon_{i}^{\nu}\left(n_{a}\right),
$$

with $d^{\mu \nu}\left(k_{i}, n_{a}\right)$ defined in (2.6). It follows simply from the transversality condition $n_{a} \cdot d\left(k_{i}, n_{a}\right)=0$. The above remark leads to a conclusion that the gauge invariant amplitude (3.4) can be written as

$$
\tilde{\mathcal{A}}\left(\varepsilon_{2}, \ldots, \varepsilon_{N}\right)=\mathcal{A}\left(\varepsilon_{2}, \ldots, \varepsilon_{i-1}, d\left(k_{i}, n_{a}\right) \cdot \varepsilon_{i}, \varepsilon_{i+1}, \ldots, \varepsilon_{N}\right)
$$

that is one can apply the transverse projector $d^{\mu \nu}\left(k_{i}, n_{a}\right)$ to any number (at least one) of external on-shell legs. In particular, one can apply the projectors to all on-shell legs. Then, (3.5) is trivially satisfied for any $i$, since $k_{i} \cdot d\left(k_{i}, n_{a}\right)=0$ for $k_{i}^{2}=0$. Thus one could have the impression that it is also possible for any gauge vector $n \neq n_{a}$, as the projector remains transverse to $k_{i}$ and the 'naive' Ward identity is satisfied. This however does not mean that such amplitude is gauge invariant — we relegate this issue to appendix C. 


\section{Analytical and numerical tests}

\subsection{Numerical tests}

We tested (3.5) numerically up to $N=12$, calculating $\mathcal{A}$ with numerical Berends-Giele recursion [37]. We also tested (3.5) for color-dressed amplitudes up to $N=8$, by calculating $\mathcal{M}$ in the color-flow representation following the method of [38, 39]. The color-dressed version of $\mathcal{W}$ was calculated by straightforward multiplication with the color-dependent factor and summation over the necessary permutations, i.e. following eq. (2.22) with the color-factor in the color-flow representation [40, 41].

\subsection{Analytic expressions for $g^{*} g \rightarrow g g$}

Let us start with the amplitude with full dependence on polarization vectors. Defining

$$
\varepsilon_{1}^{\mu}=\frac{k_{T}^{\mu}}{\left|\vec{k}_{T}\right|}
$$

we get explicitly for color-ordering $\left(a_{1}, a_{2}, a_{3}, a_{4}\right)$

$$
\begin{aligned}
\tilde{\mathcal{A}} & \left(\varepsilon_{2}, \varepsilon_{3}, \varepsilon_{4}\right)=\tilde{\mathcal{A}}\left(\varepsilon_{4}, \varepsilon_{3}, \varepsilon_{2}\right)=\frac{g^{2}}{2 k_{2} \cdot k_{3} k_{3} \cdot k_{4} k_{2} \cdot n_{a} k_{4} \cdot n_{a}} \\
& \left(n_{a} \cdot \varepsilon_{3}\left(k_{2} \cdot k_{3} k_{3} \cdot \varepsilon_{4} k_{4} \cdot n_{a} \varepsilon_{1} \cdot \varepsilon_{2}+k_{2} \cdot n_{a} k_{3} \cdot k_{4} k_{3} \cdot \varepsilon_{2} \varepsilon_{1} \cdot \varepsilon_{4}\right) k_{1}^{2}\right. \\
& +k_{4} \cdot n_{a}\left(k _ { 2 } \cdot n _ { a } \left(\left(k_{2} \cdot k_{3} k_{4} \cdot \varepsilon_{3} \mathfrak{t}-k_{3} \cdot k_{4}\left(k_{2} \cdot \varepsilon_{3} \mathfrak{r}+2 k_{2} \cdot k_{3} \varepsilon_{1} \cdot \varepsilon_{3}\right)\right) \varepsilon_{2} \cdot \varepsilon_{4}\right.\right. \\
& +k_{2} \cdot k_{3}\left(2 \left(k_{1} \cdot \varepsilon_{4} k_{2} \cdot \varepsilon_{3} \varepsilon_{1} \cdot \varepsilon_{2}-k_{1} \cdot \varepsilon_{3}\left(k_{2} \cdot \varepsilon_{4} \varepsilon_{1} \cdot \varepsilon_{2}+k_{1} \cdot \varepsilon_{2} \varepsilon_{1} \cdot \varepsilon_{4}\right)\right.\right. \\
& \left.\left.+k_{1} \cdot \varepsilon_{2}\left(k_{3} \cdot \varepsilon_{4} \varepsilon_{1} \cdot \varepsilon_{3}-k_{2} \cdot \varepsilon_{3} \varepsilon_{1} \cdot \varepsilon_{4}\right)\right)-\left(2 \mathfrak{u}_{13} \cdot \varepsilon_{2}-k_{3} \cdot \varepsilon_{2} \mathfrak{t}\right) \varepsilon_{3} \cdot \varepsilon_{4}\right) \\
& \left.+k_{3} \cdot k_{4}\left(2 k_{2} \cdot \varepsilon_{3} \mathfrak{s}_{24}+k_{3} \cdot \varepsilon_{2}\left(2 \mathfrak{s}_{34}-\mathfrak{r} \varepsilon_{3} \cdot \varepsilon_{4}\right)\right)\right) \\
& \left.+k_{2} \cdot k_{3}\left(\left(k_{4} \cdot \varepsilon_{3} n_{a} \cdot \varepsilon_{4}+k_{3} \cdot n_{a} \varepsilon_{3} \cdot \varepsilon_{4}\right) \mathfrak{p} \cdot \varepsilon_{2}-k_{1} \cdot \varepsilon_{1} k_{1} \cdot \varepsilon_{2} k_{3} \cdot \varepsilon_{4} n_{a} \cdot \varepsilon_{3}\right)\right) \\
& +k_{2} \cdot n_{a}\left(k_{3} \cdot k_{4}\left(k_{2} \cdot \varepsilon_{3} n_{a} \cdot \varepsilon_{2} \varepsilon_{1} \cdot \varepsilon_{4} k_{1}^{2}-k_{1} \cdot \varepsilon_{1} k_{1} \cdot \varepsilon_{4}\left(k_{2} \cdot \varepsilon_{3} n_{a} \cdot \varepsilon_{2}+k_{3} \cdot \varepsilon_{2} n_{a} \cdot \varepsilon_{3}\right)\right)\right. \\
& +\varepsilon_{2} \cdot \varepsilon_{3}\left(k_{4} \cdot n_{a}\left(\left(\mathfrak{t} k_{1} \cdot k_{3}-2 k_{3} \cdot k_{4} k_{3} \cdot \varepsilon_{1}\right) k_{3} \cdot \varepsilon_{4}+2 k_{3} \cdot k_{4} \mathfrak{u}_{13} \cdot \varepsilon_{4}\right)\right. \\
& \left.\left.\left.+k_{3} \cdot k_{4} k_{3} \cdot n_{a} \mathfrak{p} \cdot \varepsilon_{4}\right)\right)+k_{1} \cdot \varepsilon_{1} \varepsilon_{2} \cdot n_{a} \varepsilon_{3} \cdot n_{a} \varepsilon_{4} \cdot n_{a} k_{2} \cdot k_{3} k_{3} \cdot k_{4}\right)
\end{aligned}
$$

where we have used

$$
\begin{aligned}
\mathfrak{p}^{\alpha} & =k_{1} \cdot \varepsilon_{1} k_{1}^{\alpha}-k_{1}^{2} \varepsilon_{1}^{\alpha}, \\
\mathfrak{r} & =k_{1} \cdot \varepsilon_{1}+2 k_{2} \cdot \varepsilon_{1}-2 k_{3} \cdot \varepsilon_{1}, \\
\mathfrak{s}_{i j} & =k_{1}^{\alpha} \varepsilon_{1}^{\beta}\left(\varepsilon_{i \alpha} \varepsilon_{j \beta}-\varepsilon_{i \beta} \varepsilon_{j \alpha}\right), \\
\mathfrak{t} & =k_{1} \cdot \varepsilon_{1}+2 k_{2} \cdot \varepsilon_{1}, \\
\mathfrak{u}_{i j}^{\alpha} & =k_{i}^{\alpha} k_{j} \cdot \varepsilon_{1}-k_{i} \cdot k_{j} \varepsilon_{1}^{\alpha} .
\end{aligned}
$$

We have not used any particular reference momenta for the polarization vectors - by applying different choices the above expression can be simplified. Note, that we are allowed to play with the reference momenta as the above amplitude is gauge invariant; the $\mathcal{W}$ piece is already included above (the last term). The other color-ordered amplitudes are obtained by exchanging the relevant polarization vectors and momenta in the expression above. 
Using the spinor helicity formalism one can calculate those amplitudes explicitly for different helicity combinations. For this purpose it proves most convenient to choose $n_{a}$ as a reference vector for each of the on-shell gluons. Labeling spinors associated with the light-like momenta $n_{a}, k_{2}, k_{3}, k_{4}$ with $a, 2,3,4$ respectively, and further using the notation introduced in section 2.2 (see also [35]), we find

$$
\begin{aligned}
& \tilde{\mathcal{A}}\left(\varepsilon_{2}^{-}\left(n_{a}\right), \varepsilon_{3}^{-}\left(n_{a}\right), \varepsilon_{4}^{-}\left(n_{a}\right)\right)=0, \\
& \tilde{\mathcal{A}}\left(\varepsilon_{2}^{+}\left(n_{a}\right), \varepsilon_{3}^{+}\left(n_{a}\right), \varepsilon_{4}^{+}\left(n_{a}\right)\right)=0, \\
& \tilde{\mathcal{A}}\left(\varepsilon_{2}^{-}\left(n_{a}\right), \varepsilon_{3}^{-}\left(n_{a}\right), \varepsilon_{4}^{+}\left(n_{a}\right)\right)=\frac{g^{2}}{\sqrt{2}} \frac{\left[3\left|\not_{T}\right| a\right\rangle}{\left|\vec{k}_{T}\right|} \frac{[4 a]^{3}}{[3 a][a 2][23][34]}, \\
& \tilde{\mathcal{A}}\left(\varepsilon_{2}^{+}\left(n_{a}\right), \varepsilon_{3}^{+}\left(n_{a}\right), \varepsilon_{4}^{-}\left(n_{a}\right)\right)=\frac{g^{2}}{\sqrt{2}} \frac{\left\langle a\left|\not k_{T}\right| 3\right]}{\left|\vec{k}_{T}\right|} \frac{\langle 4 a\rangle^{3}}{\langle a 3\rangle\langle a 2\rangle\langle 23\rangle\langle 34\rangle}, \\
& \tilde{\mathcal{A}}\left(\varepsilon_{2}^{-}\left(n_{a}\right), \varepsilon_{3}^{+}\left(n_{a}\right), \varepsilon_{4}^{+}\left(n_{a}\right)\right)=\frac{g^{2}}{\sqrt{2}} \frac{\left\langle a\left|\not k_{T}\right| 3\right]}{\left|\vec{k}_{T}\right|} \frac{\langle a 2\rangle^{3}}{\langle a 3\rangle\langle 23\rangle\langle 34\rangle\langle 4 a\rangle}, \\
& \tilde{\mathcal{A}}\left(\varepsilon_{2}^{+}\left(n_{a}\right), \varepsilon_{3}^{-}\left(n_{a}\right), \varepsilon_{4}^{-}\left(n_{a}\right)\right)=\frac{g^{2}}{\sqrt{2}} \frac{\left[3\left|\not_{T}\right| a\right\rangle}{\left|\vec{k}_{T}\right|} \frac{[a 2]^{3}}{[3 a][23][34][4 a]}, \\
& \tilde{\mathcal{A}}\left(\varepsilon_{2}^{-}\left(n_{a}\right), \varepsilon_{3}^{+}\left(n_{a}\right), \varepsilon_{4}^{-}\left(n_{a}\right)\right)=\frac{g^{2}}{\sqrt{2}} \frac{\left[3\left|\not k_{T}\right| a\right\rangle}{\left|\vec{k}_{T}\right|} \frac{[3 a]^{3}}{[a 2][23][34][4 a]}, \\
& \tilde{\mathcal{A}}\left(\varepsilon_{2}^{+}\left(n_{a}\right), \varepsilon_{3}^{-}\left(n_{a}\right), \varepsilon_{4}^{+}\left(n_{a}\right)\right)=\frac{g^{2}}{\sqrt{2}} \frac{\left\langle a\left|\not k_{T}\right| 3\right]}{\left|\vec{k}_{T}\right|} \frac{\langle a 3\rangle^{3}}{\langle a 2\rangle\langle 23\rangle\langle 34\rangle\langle 4 a\rangle} .
\end{aligned}
$$

All momenta are assumed to be incoming in the formulas above. In what follows we skip the reference momenta indication in the amplitudes.

We may decompose $k_{T}^{\mu}$ in terms of $k_{3}^{\mu}$ and another light-like momentum $p^{\mu}$ following

$$
k_{T}^{\mu}=p^{\mu}+\frac{k_{T}^{2}}{2 k_{T} \cdot k_{3}} k_{3}^{\mu} .
$$

Then, we have

$$
\left|\vec{k}_{T}\right|^{2}=-k_{T}^{2}=2 \frac{\left(k_{3} \cdot p\right)\left(p \cdot n_{a}\right)}{k_{3} \cdot n_{a}},
$$

and

$$
\left|\left[3\left|\not k_{T}\right| a\right\rangle\right|^{2}=|[3|\not p| a\rangle|^{2}=4\left|k_{3} \cdot p\right|\left|p \cdot n_{a}\right|,
$$

so

$$
\left|\frac{\left[3\left|\not_{T}\right| a\right\rangle}{\left|\vec{k}_{T}\right|}\right|^{2}=4\left|k_{3} \cdot p\right|\left|p \cdot n_{a}\right| \frac{\left|k_{3} \cdot n_{a}\right|}{2\left|k_{3} \cdot p\right|\left|p \cdot n_{a}\right|}=2\left|k_{3} \cdot n_{a}\right| .
$$

Thus for the squared helicity amplitudes, we find

$$
\begin{aligned}
\left|\tilde{\mathcal{A}}\left(\varepsilon_{2}^{-}, \varepsilon_{3}^{-}, \varepsilon_{4}^{+}\right)\right|^{2} & =\left|\tilde{\mathcal{A}}\left(\varepsilon_{2}^{+}, \varepsilon_{3}^{+}, \varepsilon_{4}^{-}\right)\right|^{2}=\frac{g^{4}}{2} \frac{\left|k_{4} \cdot n_{a}\right|^{4}}{\left|n_{a} \cdot k_{2}\right|\left|k_{2} \cdot k_{3}\right|\left|k_{3} \cdot k_{4}\right|\left|k_{4} \cdot n_{a}\right|} \\
\left|\tilde{\mathcal{A}}\left(\varepsilon_{2}^{-}, \varepsilon_{3}^{+}, \varepsilon_{4}^{+}\right)\right|^{2} & =\left|\tilde{\mathcal{A}}\left(\varepsilon_{2}^{+}, \varepsilon_{3}^{-}, \varepsilon_{4}^{-}\right)\right|^{2}=\frac{g^{4}}{2} \frac{\left|k_{2} \cdot n_{a}\right|^{4}}{\left|n_{a} \cdot k_{2}\right|\left|k_{2} \cdot k_{3}\right|\left|k_{3} \cdot k_{4}\right|\left|k_{4} \cdot n_{a}\right|} \\
\left|\tilde{\mathcal{A}}\left(\varepsilon_{2}^{-}, \varepsilon_{3}^{+}, \varepsilon_{4}^{-}\right)\right|^{2} & =\left|\tilde{\mathcal{A}}\left(\varepsilon_{2}^{+}, \varepsilon_{3}^{-}, \varepsilon_{4}^{+}\right)\right|^{2}=\frac{g^{4}}{2} \frac{\left|k_{3} \cdot n_{a}\right|^{4}}{\left|n_{a} \cdot k_{2}\right|\left|k_{2} \cdot k_{3}\right|\left|k_{3} \cdot k_{4}\right|\left|k_{4} \cdot n_{a}\right|} .
\end{aligned}
$$




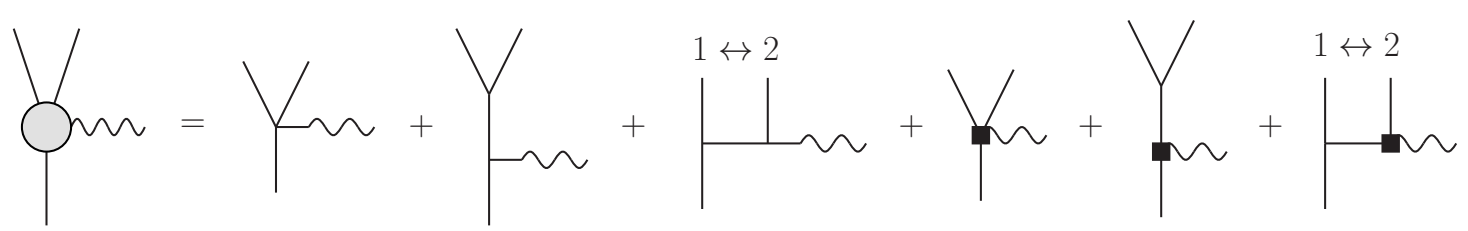

Figure 5. The effective reggeon-gluon-gluon-gluon vertex as obtained from figure 6 in [13], by expanding all blobs on the r.h.s into graphs using figure 4 in [13].

Applying eq. (2.23) we find agreement with [42]. The overall factor $\left(k_{1} \cdot k_{2} / k_{1} \cdot p_{2}\right)^{2}$ in that publication is due to a difference in the the definition of $p_{2}$ there and $n_{a}$ here.

\subsection{Comparison with Lipatov's effective action approach}

Let us now show that the gluonic amplitudes with one off-shell leg augmented with the 'gauge restoring' amplitude (3.6) are equivalent to the amplitudes obtained using the effective reggeon-gluon vertices of [13]. In the vertices, no external gluon is necessarily on-shell, and the reggeon essentially is an off-shell gluon with momentum $k_{1}^{\mu}=E\left(n^{+}\right)^{\mu}+k_{T}^{\mu}$, where $\left(n^{+}\right)^{\mu}=n_{a}^{\mu} / E$. If all gluons, except the reggeon, are on-shell, the vertices correspond to our amplitudes, modulo a factor $E /\left|\vec{k}_{T}\right|$.

This can most easily be understood considering figure 5. It graphically depicts the reggeon-gluon-gluon-gluon vertex as presented in [13]. Straight lines are gluons, and the wavy line attached to a straight line is the off-shell gluon without a propagator, but contracted with $\left|\vec{k}_{T}\right|^{2}\left(n^{+}\right)^{\mu}=\left(\left|\vec{k}_{T}\right| / E\right) \mathfrak{e}_{1}^{\mu}$. Here seems to be a difference with our approach, in which $\mathfrak{e}_{1}^{\mu}$ is contracted to the off-shell leg including the propagator, and in particular including the projector in the axial gauge. Notice, however, that the collection of all graphs with a wavy line attached to a straight line is exactly the set of usual Feynman graphs, calculated with usual Feynman rules, and before contracting them with $\mathfrak{e}_{1}^{\mu}$ their sum forms an off-shell current $J_{1}^{\mu}$, satisfying current conservation

$$
k_{1} \cdot J_{1}=0 .
$$

The off-shell current is not gauge-invariant, but eq. (4.23) holds in any gauge. Consequently, we have

$$
\mathfrak{e}_{1}^{\mu} d_{\mu \nu}\left(k_{1}, n\right) J_{1}^{\nu}=\mathfrak{e}_{1} \cdot J_{1}-\frac{\mathfrak{e}_{1} \cdot k_{1}}{n \cdot k_{1}} n \cdot J_{1}=\mathfrak{e}_{1} \cdot J_{1},
$$

where the second equality follows from the fact that $\mathfrak{e}_{1} \cdot k_{1}=\left|\vec{k}_{1 T}\right| n_{a} \cdot k_{1}=0$. So we see that it does not matter for the off-shell external line whether the projector is present or not, in any gauge.

The vertices in figure 5 indicated by a square represent the effective vertices of equation (18) in [13], which have the essential feature that all gluons attached to it are contracted with $\left(n^{+}\right)^{\mu}$. Since the results of [13] are gauge-invariant, the graphs can be calculated in the axial gauge with gauge-vector $\left(n^{+}\right)^{\mu}$, with the consequence that the last two graphs vanish, since they contain a gluon propagator attached to the square vertex. The fourth graph on the r.h.s. stays, and can readily be recognized as our 'gauge restoring' amplitude (3.6). 
The other graphs are the usual graphs one would take into account in a fully on-shell calculation, and are calculated following the usual Feynman rules. The same argumentation holds for vertices with arbitrary number of on-shell gluons. All graphs containing square vertices vanish, except one which corresponds to (3.6), and the other graphs are the usual ones taken into account in a fully on-shell calculation.

\section{$5 \quad$ Summary and outlook}

We provided a prescription to calculate gauge-invariant multi-gluon scattering amplitudes within high energy factorization, for which one initial state gluon is off-shell. Gaugeinvariance was ensured by employing Slavnov-Taylor identities, enabling the construction of the necessary extra contributions to the amplitude besides those coming from the usual Feynman graphs. In particular, we presented compact expressions for the helicity amplitudes of the process $g^{*} g \rightarrow g g$, and observed that they are in agreement with existing calculations. Also, we found agreement of our prescription with the effective action approach of Lipatov. The generalization to two off-shell legs is currently under study.

\section{Acknowledgments}

The authors are grateful to F. Hautmann for extensive discussions and reading the preceding notes. P.K. would like to thank also H. Arodz, L. Motyka, W. Slominski and L. Szymanowski. We also acknowledge useful discussions with M. Deak and H. Jung.

During this research P.K. and K.K have been supported by NCBiR grant LIDER/02/35/L-2/10/NCBiR/2011.

\section{A Ghosts and Slavnov-Taylor identities}

Let us consider the pure Yang-Mills Lagrangian with gauge fixing and ghost terms

$$
\mathcal{L}_{\mathrm{YM}}=-\frac{1}{4} F_{\mu \nu}^{a} F_{a}^{\mu \nu}-\frac{1}{2 \lambda} \mathcal{F}_{a}^{2}(A)+\bar{c}_{a} \frac{\delta \mathcal{F}_{a}(A)}{\delta A_{\mu}^{c}} D_{\mu}^{c b} c_{b}
$$

where $A_{a}^{\mu}$ is a gluon field, $c_{a}, \bar{c}_{a}$ represent ghost and anti-ghost fields, further $F_{a}^{\mu \nu}=$ $\partial^{\mu} A_{a}^{\nu}-\partial^{\nu} A_{a}^{\mu}+g f_{a b c} A_{b}^{\mu} A_{c}^{\nu}$ and $D_{\mu}^{a b}=\delta^{a b} \partial_{\mu}+g f_{a b c} A_{\mu}^{c}$. Here $f_{a b c}$ are the usual $\mathrm{SU}\left(N_{c}\right)$ structure constants. We consider two choices of the gauge-fixing function

$$
\begin{array}{ll}
\mathcal{F}_{a}(A)=\partial_{\mu} A_{a}^{\mu} & (\text { covariant gauge }) \\
\mathcal{F}_{a}(A)=n_{\mu} A_{a}^{\mu} & \text { (general axial gauge })
\end{array}
$$

where in general $n^{2} \neq 0$. There is still a residual freedom in the choice of $\lambda$ parameter which can be utilized in order to simplify calculations.

Given the Lagrangian above, one can obtain the Feynman rules for gluons and ghosts, see appendix B. 
It is usually argued that the ghosts decouple from the theory in the axial gauge defined by $\lambda \rightarrow 0$ [36]. Indeed, any internal gluon line is attached to a ghost line following

$$
n^{\mu} D_{\mu \nu}(k, n)=-i \lambda \frac{k_{\nu}}{k \cdot n}
$$

and thus vanishes for $\lambda \rightarrow 0$. The only candidates for non-vanishing graphs with closed ghost loops are of the type

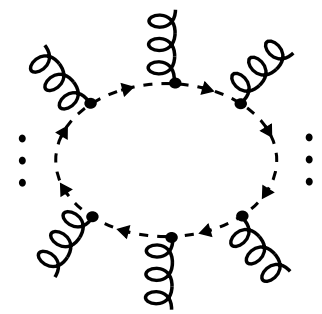

where all gluons are external. Using the Feynman rules and dimensional regularization for the loop integral we have (modulo couplings and phase factors)

$$
I_{\text {gh-loop }}=\int \frac{d^{D} k}{k \cdot n\left(k+p_{1}\right) \cdot n \ldots\left(k+p_{1}+\ldots p_{M}\right) \cdot n},
$$

where $p_{1}, \ldots, p_{M}$ are the momenta of the gluons. Introducing Feynman parameters and shifting the integration variable we get

$$
I_{\text {gh-loop }}=\int_{0}^{1} \prod_{i=1}^{M} d x_{i} \int \frac{d^{D} k \delta\left(1-x_{1}-\ldots-x_{M}\right)}{(k \cdot n)^{M}}=\int \frac{d^{D} k}{(k \cdot n)^{M}}=0
$$

in dimensional regularization, so also these graphs vanish. In certain applications it is however convenient to consider also open ghost lines, and indeed the following graphs

$$
22 \ldots \varepsilon
$$

in which all gluons are external do not necessarily vanish. This is precisely what is used in the present paper.

The Yang-Mills action $\mathcal{L}_{\mathrm{YM}}$ is invariant under the Becchi-Rouet-Stora-Tyutin (BRST) transformation (see e.g. [43] for a review). The resulting Slavnov-Taylor identity for a functional for connected Green functions $W$ reads

$$
\int d^{4} x\left\{j_{\mu}^{a}(x) \frac{\delta}{\delta K_{\mu}^{a}(x)}-\bar{\xi}_{a}(x) \frac{\delta}{\delta H_{a}(x)}+\xi_{a}(x) F^{a}\left(\frac{\delta}{\delta j(x)}\right)\right\} W[j, \xi, \bar{\xi}, H, K]=0,
$$

where $j_{\mu}^{a}, \bar{\xi}_{a}, \xi_{a}$ are the sources for gluons, ghosts and anti-ghosts respectively. The sources $K_{\mu}^{a}$ and $H_{a}$ generate BRST terms $\mathcal{K}_{\mu}^{a}$ and $\mathcal{H}_{a}$ respectively, where

$$
\begin{aligned}
\mathcal{K}_{\mu}^{a} & =\lambda\left(\partial_{\mu} c^{a}(x)-g f_{a c d} A_{\mu}^{c}(x) c^{d}(x)\right), \\
\mathcal{H}_{a} & =\frac{1}{2} \lambda g f_{a d e} c^{d}(x) c^{e}(x) .
\end{aligned}
$$


The identities for the Green functions are derived by differentiating eq. (A.9) with respect to various sources. For instance, the identity in figure $2 \mathrm{~A}$ is obtained by applying the following functional derivative

$$
\frac{\delta^{n}}{\delta j_{\mu_{n}}^{a_{n}}\left(x_{n}\right) \ldots \delta j_{\mu_{3}}^{a_{3}}\left(x_{3}\right) \delta \xi_{a_{2}}\left(x_{2}\right) \delta j_{\mu_{1}}^{a_{1}\left(x_{1}\right)}}\left[\text { eq. (A.9)] }\left.\right|_{j_{\mu}^{a}=\bar{\xi}_{a}=\xi_{a}=K_{\mu}^{a}=H_{a}=0} .\right.
$$

The various indices $x_{i}, a_{i}, \mu_{i}$ correspond to a position, color and Lorentz index for a $\operatorname{leg} i$. Let us note, that depending on the form of the gauge-fixing function $F(A)$, the resulting identities involve either the derivative of the gluon field (in covariant gauge) or contraction with the gauge vector $n$ (in axial gauge). The former leads in momentum space to contraction with the corresponding gluon momentum.

\section{B Color-ordered Feynman rules}

For the reader's convenience we gather the Feynman rules for gluons and ghosts in covariant and general axial gauges. The more customary Feynman and pure axial gauge are obtained by setting $\lambda=1$ and $\lambda=0$ respectively.

- for a given color ordering of external legs one writes only planar diagrams

- gluon propagator

$$
\mu w^{\nu}= \begin{cases}\frac{-i}{k^{2}+i \varepsilon}\left[\eta^{\mu \nu}+(\lambda-1) \frac{k^{\mu} k^{\nu}}{k^{2}}\right] & \text { (covariant gauge) } \\ \frac{-i}{k^{2}+i \varepsilon}\left[d^{\mu \nu}(k, n)+\lambda k^{2} \frac{k^{\mu} k^{\nu}}{(k \cdot n)^{2}}\right] & \text { (general axial gauge) }\end{cases}
$$

- ghost propagator (momentum flows right)

$$
\bullet \cdots-\cdots= \begin{cases}\frac{-i}{k^{2}+i \epsilon} & (\text { covariant gauge }) \\ \frac{-i}{k \cdot n+i \epsilon} & \text { (general axial gauge) }\end{cases}
$$

- triple gluon vertex (all momenta are outgoing)

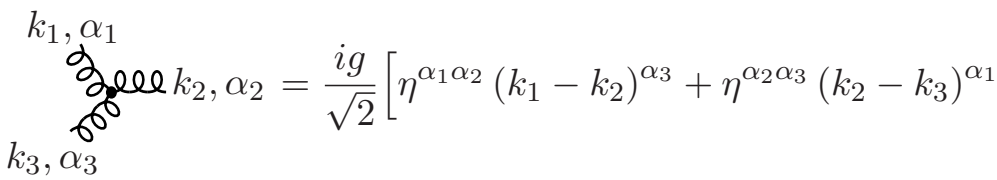

$$
\begin{aligned}
& \left.+\eta^{\alpha_{3} \alpha_{1}}\left(k_{3}-k_{1}\right)^{\alpha_{2}}\right]
\end{aligned}
$$


- quartic gluon vertex

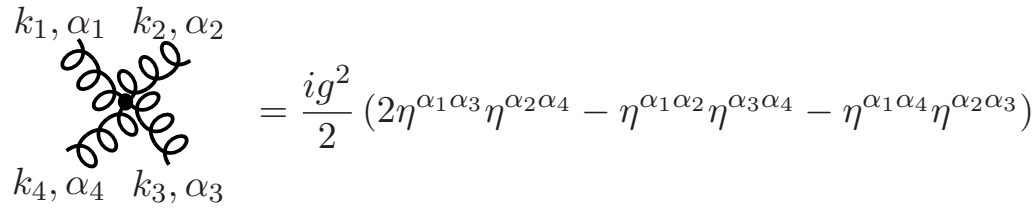

- ghost-gluon vertex

$$
\begin{array}{ll}
k_{1} \\
k_{3}
\end{array}
$$

Let us also give some auxiliary rules that are used in order to present the SlavnovTaylor identities (since we use color-ordered rules the color indices are skipped):

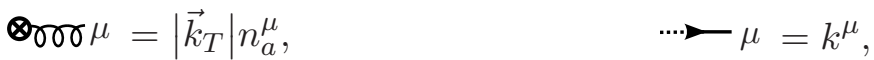

$$
\begin{aligned}
& \cdots \triangleright \mu=n^{\mu}, \quad \quad \#=d^{\mu \nu}(k, n) .
\end{aligned}
$$

The color-ordered Feynman rule for the BRST vertex originating in second term of eq. (A.10) is

$$
\underset{\mu}{\$} \boldsymbol{*}=\frac{g}{\sqrt{2}} n_{a}^{\mu} \text {. }
$$

\section{Axial gauge and external projectors}

It was argued in the end of section 3.1 that the gauge invariant amplitude $\tilde{\mathcal{A}}$ can be obtained by applying at least one projector $d^{\mu \nu}\left(k_{i}, n_{a}\right)$ to the external on-shell legs, cf. eq. (3.18). Recall that it applies in the axial gauge with $n_{a}$ taken as the gauge vector.

One can wonder, whether the following amplitude

$$
\tilde{\mathcal{A}}^{\prime}\left(\varepsilon_{2}, \ldots, \varepsilon_{N}\right)=\mathcal{A}\left(d\left(k_{2}, n\right) \cdot \varepsilon_{2}, \ldots, d\left(k_{N}, n\right) \cdot \varepsilon_{N}\right),
$$

which satisfies

$$
\tilde{\mathcal{A}}^{\prime}\left(\varepsilon_{2}, \ldots, \varepsilon_{i-1}, k_{i}, \varepsilon_{i+1}, \ldots, \varepsilon_{N}\right)=0
$$

for any $i$ by definition also when $n \neq n_{a}$, is gauge invariant. Below, we argue that it is not the case. 
The point is, that although the identity (C.2) is satisfied, the true Ward identity is not fulfilled, unless $n=n_{a}$. Actually, the S-T identities that are relevant here involve contraction with the gauge vector $n$, not with the momentum of the corresponding line (appendix A).

To be more specific, consider the corresponding Green function, this time fully in the axial gauge (including the external on-shell lines) with the gauge vector $n$. At this stage we have to keep the dependence on the gauge parameter $\lambda$. After applying the reduction formula (3.1) it reduces to the amplitude $\tilde{\mathcal{A}}^{\prime}$. As remarked above, the S-T identity is realized here by contracting one of the external lines (say $k_{2}$ ) with $n$. Thus we have diagramatically for the Green function (using the auxiliary Feynman rules from appendix B)

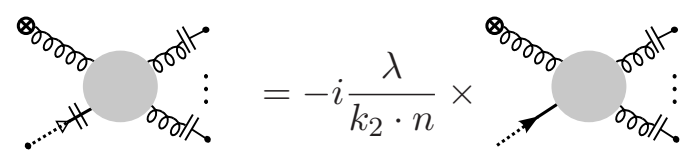

due to eq. (A.4). Note, that there is no external propagator for $k_{2}$ on the r.h.s. (it is indicated by the lack of a dot at the end of the propagator). On the other hand, applying the S-T identity to the l.h.s. of this relation we get

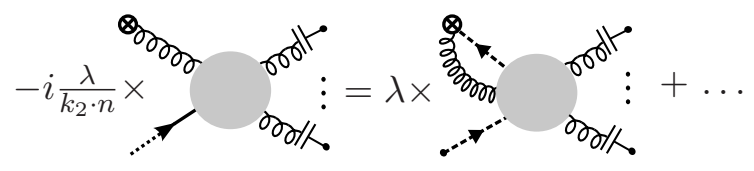

where the horizontal dots denote the rest of possible gauge terms, similar to those in figure $2 \mathrm{~A}$, but all of them are multiplied by $\lambda$ according to (A.10). Now, we apply the reduction formula to this identity. Let us note, that there appears the factor $\lambda k_{2}^{2} / k_{2} \cdot n$ for each term ( $k_{2}^{2}$ is not cancelled on the r.h.s. as the external propagator is $1 / k_{2} \cdot n$ there), thus it can be omitted. Since $\lambda$ cancelled, we may now set $\lambda=0$. The final form of the S-T identity is (up to a phase factor)

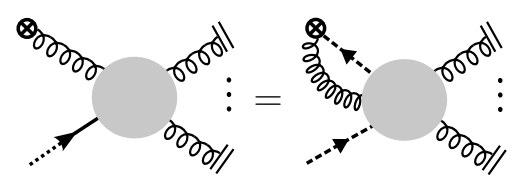

which looks the same as figure $2 \mathrm{~B}$ with however the external projectors inserted for the on-shell gluons.

It means that the correct identity for the amplitude with the external projectors which is connected with the local gauge invariance is the one above, not (C.2). Moreover, if one wants to have zero on the r.h.s. the gauge vector has to be equal to $n_{a}$. This is because the amplitude on the r.h.s. has the form

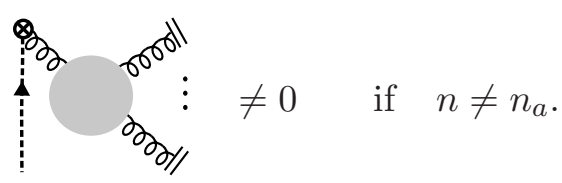


The other diagrams are zero, because here both the internal and the external lines cannot be attached to the ghost line; the only possibility is the diagram above. It is nonzero, because the eikonal vertex is proportional to $n_{a}$, while $n_{a}^{\mu} d_{\mu \nu}(k, n) \neq 0$.

Let us finally remark, that the relation (C.5) justifies our assumption that one can use different gauges for on-shell and off-shell lines in order to analyze the diagrams (see also the comment under (C.5)).

\section{Proof of the 'gauge restoring' amplitude $\mathcal{W}$}

In the following we shall justify the equation (3.6) for any number of gluons.

The proof consist in two steps. First, let us argue that

$$
\tilde{\mathcal{A}}\left(\varepsilon_{2}, \ldots, \varepsilon_{N}\right)=\mathcal{A}\left(\varepsilon_{2}, \ldots, \varepsilon_{N}\right)-\mathcal{A}\left(k_{2}, \ldots, k_{N}\right) \alpha_{2} \ldots \alpha_{N}
$$

where

$$
\alpha_{i}=\frac{\varepsilon_{i} \cdot n_{a}}{k_{i} \cdot n_{a}}
$$

To this end let us show that $\tilde{\mathcal{A}}\left(\varepsilon_{2}, \ldots, \varepsilon_{i-1}, k_{i}, \varepsilon_{i+1}, \ldots, \varepsilon_{N}\right)=0$. To be specific set $i=2$, i.e. replace $\varepsilon_{2}$ by $k_{2}$. Graphically, we have (see appendix B for the auxiliary Feynman rules)

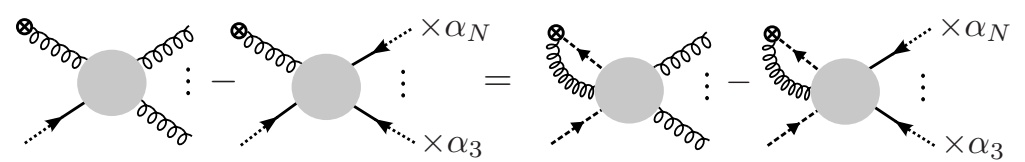

where we have used S-T identities on the r.h.s. (the rest of the terms are zero, see the discussion to figure 2). Note next, that since we use the axial gauge with $n_{a}$ as the gauge vector for internal lines, only an external gluon line can couple to a ghost line or eikonal vertex, both of which couple via $n_{a}^{\mu}$. Therefore we actually get zero on the r.h.s: for the first diagram we get the factors $\varepsilon_{i} \cdot n_{a}$ for each leg, while for the second diagram we get $k_{i} \cdot n_{i} \alpha_{i}=\varepsilon_{i} \cdot n_{a}$, thus the result is indeed zero.

What remains is to prove that

$$
\mathcal{A}\left(k_{2}, \ldots, k_{N}\right) \alpha_{2} \ldots \alpha_{n}=-\mathcal{W}\left(\varepsilon_{2}, \ldots, \varepsilon_{N}\right) .
$$

It is accomplished, by noting that

$$
\mathcal{A}\left(k_{2}, \ldots, k_{N}\right)=\left(-\frac{g}{\sqrt{2}}\right)^{N-2}\left|\vec{k}_{T}\right| \frac{k_{3} \cdot n_{a} \ldots k_{N} \cdot n_{a}}{\left(k_{2}-k_{3}\right) \cdot n_{a} \ldots\left(k_{2}-\ldots-k_{N-1}\right) \cdot n_{a}}
$$

as is evident from the following diagrammatic expression

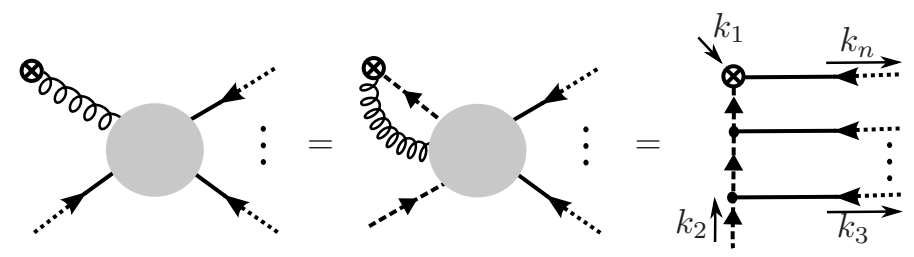

where we have used the S-T identity with respect to the leg with momentum $k_{2}$. Thus, $\mathcal{A}\left(k_{2}, \ldots, k_{N}\right)$ multiplied by $\alpha_{2} \ldots \alpha_{N}$ indeed recovers (3.6). 


\section{E Singular behaviour of the amplitude $\tilde{\mathcal{A}}$}

Let us show, that the gauge invariant off-shell amplitude $\tilde{\mathcal{A}}=\mathcal{A}+\mathcal{W}$ possesses the correct singular behaviour in the collinear and soft limits. For the purpose of this part let us supply the amplitudes with an additional subscript denoting the number of legs, thus we write $\tilde{\mathcal{A}} \equiv \tilde{\mathcal{A}}_{N}, \mathcal{A} \equiv \mathcal{A}_{N}, \mathcal{W} \equiv \mathcal{W}_{N}$, if there are $N$ external legs.

First, let us investigate the collinear limit. Let us start with analyzing the corresponding limit of the gauge non-invariant amplitude $\mathcal{A}$. To this end consider the setup as in the figure below

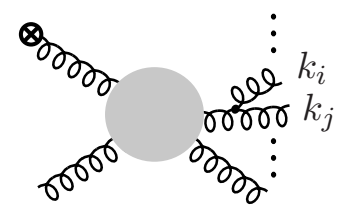

where the momenta $k_{i}, k_{j}$ are parametrized as

$$
\begin{aligned}
& k_{i}^{\mu}=z p^{\mu}+\frac{\left|\vec{p}_{T}\right|^{2}}{2 z p \cdot n_{a}} n_{a}^{\mu}+p_{T}^{\mu}, \\
& k_{j}^{\mu}=(1-z) p^{\mu}+\frac{\left|\vec{p}_{T}\right|^{2}}{2(1-z) p \cdot n_{a}} n_{a}^{\mu}-p_{T}^{\mu},
\end{aligned}
$$

with $p^{2}=0$ and $p_{T}^{2}=-\left|\vec{p}_{T}\right|^{2}$. With this decomposition of the momenta the scalar part of the gluon propagator reads

$$
\frac{1}{\left(k_{i}+k_{j}\right)^{2}}=\frac{1}{\langle i j\rangle[j i]}=\frac{z(1-z)}{\left|\vec{p}_{T}\right|^{2}} .
$$

The collinear limit is approached when

$$
\left|\vec{p}_{T}\right| \rightarrow 0
$$

Then $k_{i}=z p, k_{j}=(1-z) p$ and $k_{i}+k_{j}=p$. Let us also note the following usefull identities

$$
\begin{aligned}
& \varepsilon_{i}^{\left(\lambda_{i}\right)} \cdot p=-\frac{1}{z}\left(\frac{\left|\vec{p}_{T}\right|^{2}}{2 z p^{+}} \varepsilon_{i}^{\left(\lambda_{i}\right)} \cdot n_{a}+\varepsilon_{i}^{\left(\lambda_{i}\right)} \cdot p_{T}\right), \\
& \varepsilon_{j}^{\left(\lambda_{j}\right)} \cdot p=-\frac{1}{1-z}\left(\frac{\left|\vec{p}_{T}\right|^{2}}{2(1-z) p^{+}} \varepsilon_{j}^{\left(\lambda_{j}\right)} \cdot n_{a}-\varepsilon_{j}^{\left(\lambda_{j}\right)} \cdot p_{T}\right),
\end{aligned}
$$

which follow directly from the decompositions (E.2), (E.3) and ortogonality relation (2.11).

In the collinear limit the amplitude behaves as

$$
\mathcal{A}_{N}\left(\varepsilon_{2}, \ldots, \varepsilon_{i}^{\left(\lambda_{i}\right)}, \varepsilon_{j}^{\left(\lambda_{j}\right)}, \ldots, \varepsilon_{N}\right) \underset{k_{i} \| k_{j}}{\longrightarrow} \mathcal{A}_{N-1}\left(\varepsilon_{2}, \ldots, \mathfrak{J}_{p}^{\left(\lambda_{i}, \lambda_{j}\right)}, \ldots, \varepsilon_{N}\right),
$$

where the 'collinear current' $\mathfrak{J}_{p}$ depends on the helicities $\lambda_{i}, \lambda_{j}$ of the gluons $i, j$ and is defined as

$$
\mathfrak{J}_{p}^{\left(\lambda_{i}, \lambda_{j}\right) \mu}=\lim _{\left|\vec{p}_{T}\right| \rightarrow 0} \mathfrak{J}^{\left(\lambda_{i}, \lambda_{j}\right) \mu}\left(k_{i}, k_{j}\right)
$$


where

$$
\mathfrak{J}^{\left(\lambda_{i}, \lambda_{j}\right) \mu}\left(k_{i}, k_{j}\right)=\frac{-i d^{\mu \nu}\left(k_{i}+k_{j}\right)}{\langle i j\rangle[j i]} V_{3 \nu \alpha_{i} \alpha_{j}}\left(-k_{i}-k_{j}, k_{i}, k_{j}\right) \varepsilon_{i}^{\left(\lambda_{i}\right) \alpha_{i}} \varepsilon_{j}^{\left(\lambda_{j}\right) \alpha_{j}} .
$$

In the equation above we have denoted the three-point gluon vertex as $V_{3}^{\alpha_{1} \alpha_{2} \alpha_{3}}\left(q_{1}, q_{2}, q_{3}\right)$ (see appendix B), while the projection tensor is defined in eq. (2.6).

We obtain

$$
\begin{aligned}
\mathfrak{I}^{\left(\lambda_{i}, \lambda_{j}\right) \mu}\left(k_{i}, k_{j}\right)= & \frac{\sqrt{2} g}{\langle i j\rangle[j i]} \\
& \left\{\frac{1}{1-z}\left[\varepsilon_{i}^{\left(\lambda_{i}\right) \mu}-p^{\mu} \frac{\varepsilon_{i}^{\left(\lambda_{i}\right)} \cdot n_{a}}{p \cdot n_{a}}\right] \varepsilon_{j}^{\left(\lambda_{j}\right)} \cdot p_{T}\right. \\
& +\frac{1}{z}\left[\varepsilon_{j}^{\left(\lambda_{j}\right) \mu}-p^{\mu} \frac{\varepsilon_{j}^{\left(\lambda_{j}\right)} \cdot n_{a}}{p \cdot n_{a}}\right] \varepsilon_{i}^{\left(\lambda_{i}\right)} \cdot p_{T} \\
& \left.-p_{T}^{\mu} \varepsilon_{i}^{\left(\lambda_{i}\right)} \cdot \varepsilon_{j}^{\left(\lambda_{j}\right)}+\mathcal{O}\left(\left|\vec{p}_{T}\right|^{2}\right)\right\}
\end{aligned}
$$

where we have used eqs. (E.6), (E.7) in order to keep track of the leading terms. In the considered limit

$$
\varepsilon_{l}^{\left(\lambda_{l}\right)} \equiv \varepsilon_{k_{l}}^{\left(\lambda_{l}\right)}=\varepsilon_{p}^{\left(\lambda_{l}\right)}+\mathcal{O}\left(\left|\vec{p}_{T}\right|\right), \quad l=i, j .
$$

Now, one has to fix the helicities of the gluons. For the case $\lambda_{i}=+, \lambda_{j}=+$ we get the leading term

$$
\mathfrak{J}^{(+,+) \mu}\left(k_{i}, k_{j}\right)=\sqrt{2} g\left(\varepsilon_{p}^{+\mu}-p^{\mu} \varepsilon_{p}^{+} \cdot n_{a}\right) \frac{\varepsilon_{p}^{+} \cdot p_{T}}{z(1-z)\langle i j\rangle[j i]}+\mathcal{O}\left(\left|\vec{p}_{T}\right|^{0}\right) .
$$

In order to estimate the product $\varepsilon_{p}^{+} \cdot p_{T}$ let us calculate first $\varepsilon_{p}^{+} \cdot p$ for some reference momentum $q$ and use (E.6). We get

$$
\begin{aligned}
z \varepsilon_{k_{i}}^{+}(q) \cdot p=\frac{z\left\langle q-|\not p| k_{i}-\right\rangle}{\sqrt{2}\left\langle q k_{i}\right\rangle} & =\frac{z}{\sqrt{2}} \frac{\langle q p\rangle\left[p k_{i}\right]}{\left\langle q k_{i}\right\rangle} \\
& =\frac{z}{\sqrt{2}} \frac{q \perp \sqrt{\frac{p \cdot n_{a}}{q \cdot n_{a}}} p_{\perp}^{*} z^{-\frac{1}{2}}}{q_{\perp} \sqrt{\frac{z p \cdot n_{a}}{q \cdot n_{a}}}-p_{\perp} \sqrt{\frac{q \cdot n_{a}}{z p \cdot n_{a}}}}=\frac{p_{\perp}^{*}}{\sqrt{2}}+\mathcal{O}\left(\left|\vec{p}_{T}\right|^{2}\right)
\end{aligned}
$$

and thus

$$
\varepsilon_{p}^{+} \cdot p_{T}=\frac{p_{\perp}^{*}}{\sqrt{2}}
$$

However

$$
[i j]=-\frac{p_{\perp}^{*}}{\sqrt{z(1-z)}}, \quad\langle i j\rangle=\frac{p_{\perp}}{\sqrt{z(1-z)}}
$$

so eq. (E.13) can be written as

$$
\mathfrak{J}^{(+,+) \mu}\left(k_{i}, k_{j}\right)=g\left(\varepsilon_{p}^{+\mu}-p^{\mu} \frac{\varepsilon_{p}^{+} \cdot n_{a}}{p \cdot n_{a}}\right) \frac{1}{\sqrt{z(1-z)}\langle i j\rangle}+\mathcal{O}\left(\left|\vec{p}_{T}\right|^{0}\right) .
$$


Similarily

$$
\mathfrak{J}^{(-,-) \mu}\left(k_{i}, k_{j}\right)=g\left(\varepsilon_{p}^{-\mu}-p^{\mu} \frac{\varepsilon_{p}^{-} \cdot n_{a}}{p \cdot n_{a}}\right) \frac{1}{\sqrt{z(1-z)}[j i]}+\mathcal{O}\left(\left|\vec{p}_{T}\right|^{0}\right) .
$$

Now, consider the following helicity combination $\lambda_{i}=+, \lambda_{j}=-$. We gain an additional contribution which was not present in the previous cases, namely the one due to the last term in (E.11). In order to include this, let us decompose $p_{T}^{\mu}$ as follows

$$
p_{T}^{\mu}=\varepsilon_{p}^{+} \cdot p_{T}\left(\varepsilon_{p}^{-\mu}-p^{\mu} \frac{\varepsilon_{p}^{-} \cdot n_{a}}{p \cdot n_{a}}\right)+\varepsilon_{p}^{-} \cdot p_{T}\left(\varepsilon_{p}^{+\mu}-p^{\mu} \frac{\varepsilon_{p}^{+} \cdot n_{a}}{p \cdot n_{a}}\right) .
$$

Let us note that $\left(p_{T}^{\mu}\right)^{*}=p_{T}^{\mu}$ and $p_{T}^{2}=-2 \varepsilon_{p}^{+} \cdot p_{T} \varepsilon_{p}^{-} \cdot p_{T}$ what gives $-\left|\vec{p}_{T}\right|^{2}$ due to (E.15) and the fact that $\hat{p}_{T} \hat{p}_{T}^{*}=\left|\vec{p}_{T}\right|^{2}$. Thus it is the correct representation. Therefore

$$
\begin{aligned}
\mathfrak{J}^{(+,-) \mu}\left(k_{i}, k_{j}\right)= & \frac{\sqrt{2} g}{\langle i j\rangle[j i]}\left\{\frac{z}{1-z}\left(\varepsilon_{i}^{+\mu}-p^{\mu} \frac{\varepsilon_{i}^{+} \cdot n_{a}}{p \cdot n_{a}}\right) \varepsilon_{j}^{-} \cdot p_{T}\right. \\
& \left.+\frac{1-z}{z}\left(\varepsilon_{j}^{-\mu}-p^{\mu} \frac{\varepsilon_{j}^{-} \cdot n_{a}}{p \cdot n_{a}}\right) \varepsilon_{i}^{+} \cdot p_{T}+\mathcal{O}\left(\left|\vec{p}_{T}\right|^{2}\right)\right\} \\
= & g\left(\varepsilon_{p}^{+\mu}-p^{\mu} \frac{\varepsilon_{p}^{+} \cdot n_{a}}{p \cdot n_{a}}\right) \frac{z^{2}}{\sqrt{z(1-z)}[j i]} \\
& +g\left(\varepsilon_{p}^{-\mu}-p^{\mu} \frac{\varepsilon_{p}^{-} \cdot n_{a}}{p \cdot n_{a}}\right) \frac{(1-z)^{2}}{\sqrt{z(1-z)}\langle i j\rangle}+\mathcal{O}\left(\left|\vec{p}_{T}\right|^{0}\right) .
\end{aligned}
$$

For $\lambda_{i}=-, \lambda_{j}=+$ one has to replace $\varepsilon_{p}^{+} \longleftrightarrow \varepsilon_{p}^{-}$and $\langle i j\rangle \longleftrightarrow[j i]$. Let us note, that the coefficients of vectors in eqs. (E.17), (E.18), (E.20) are precisely the $g \rightarrow g g$ splitting amplitudes of refs. $[29,44]$. Let us denote them $P_{g g}^{\left(\lambda_{p}, \lambda_{i}, \lambda_{j}\right)}$, where $\lambda_{p}$ is the helicity of the gluon with momentum $p$. Since the 'collinear current' has a component proportional to $p^{\mu}$ the actual factorization does not occur for the amplitude $\mathcal{A}_{N}\left(\varepsilon_{2}, \ldots, \varepsilon_{N}\right)$, i.e. we have

$$
\begin{aligned}
\mathcal{A}_{N}\left(\varepsilon_{2}, \ldots \varepsilon_{i}^{\left(\lambda_{i}\right)}, \varepsilon_{j}^{\left(\lambda_{j}\right)}, \ldots, \varepsilon_{N}\right) \underset{k_{i} \| k_{j}}{\longrightarrow}\left[\mathcal{A}_{N-1}\left(\varepsilon_{2}, \ldots, \varepsilon_{p}^{\left(\lambda_{p}\right)}, \ldots, \varepsilon_{N}\right)\right. \\
\left.-\mathcal{A}_{N-1}\left(\varepsilon_{2}, \ldots, p, \ldots, \varepsilon_{N}\right) \frac{\varepsilon_{p}^{\left(\lambda_{p}\right)} \cdot n_{a}}{p \cdot n_{a}}\right] P_{g g}^{\left(\lambda_{p}, \lambda_{i}, \lambda_{j}\right)}
\end{aligned}
$$

with

$$
\mathcal{A}_{N-1}\left(\varepsilon_{2}, \ldots, p, \ldots, \varepsilon_{N}\right) \neq 0
$$

due to the off-shell leg. However, as shown in the present paper the amount of violation of gauge invariance is given by the eikonal emissions $\mathcal{W}_{N-1}$, according to eq

$$
\mathcal{A}_{N-1}\left(\varepsilon_{2}, \ldots, p, \ldots, \varepsilon_{N}\right)=-\mathcal{W}_{N-1}\left(\varepsilon_{2}, \ldots, p, \ldots, \varepsilon_{N}\right) .
$$

Noticing that

$$
\mathcal{W}_{N-1}\left(\varepsilon_{2}, \ldots, p, \ldots, \varepsilon_{N}\right) \frac{\varepsilon_{p} \cdot n_{a}}{p \cdot n_{a}}=\mathcal{W}_{N-1}\left(\varepsilon_{2}, \ldots, \varepsilon_{p}, \ldots, \varepsilon_{N}\right)
$$


we get

$$
\mathcal{A}_{N}\left(\varepsilon_{2}, \ldots \varepsilon_{i}^{\left(\lambda_{i}\right)}, \varepsilon_{j}^{\left(\lambda_{j}\right)}, \ldots, \varepsilon_{N}\right) \underset{k_{i} \| k_{j}}{\longrightarrow} \tilde{\mathcal{A}}_{N-1}\left(\varepsilon_{2}, \ldots, \varepsilon_{p}^{\left(\lambda_{p}\right)}, \ldots, \varepsilon_{N}\right) P_{g g}^{\left(\lambda_{p}, \lambda_{i}, \lambda_{j}\right)} .
$$

However

$$
\tilde{\mathcal{A}}_{N}=\mathcal{A}_{N}+\mathcal{O}\left(\left|\vec{p}_{T}\right|^{0}\right)
$$

since $\mathcal{W}_{N}$ is non-singular. Therefore

$$
\tilde{\mathcal{A}}_{N}\left(\varepsilon_{2}, \ldots \varepsilon_{i}^{\left(\lambda_{i}\right)}, \varepsilon_{j}^{\left(\lambda_{j}\right)}, \ldots, \varepsilon_{N}\right) \underset{k_{i} \| k_{j}}{\longrightarrow} \tilde{\mathcal{A}}_{N-1}\left(\varepsilon_{2}, \ldots, \varepsilon_{p}^{\left(\lambda_{p}\right)}, \ldots, \varepsilon_{N}\right) P_{g g}^{\left(\lambda_{p}, \lambda_{i}, \lambda_{j}\right)}
$$

i.e. the amplitude $\tilde{\mathcal{A}}_{N}$ posseses the correct collinear behaviour.

Let us now turn to the soft limit. It is approached when the momentum of a gluon vanishes, i.e.

$$
k_{i}^{\mu}=\lambda r^{\mu}, \lambda \rightarrow 0
$$

for any fixed light-like four-vector $r^{\mu}$. In this limit the gauge non-invariant amplitude behaves as

$$
\mathcal{A}_{N}\left(\varepsilon_{2}, \ldots, \varepsilon_{i}^{\left(\lambda_{i}\right)}, \varepsilon_{j}^{\left(\lambda_{j}\right)}, \ldots, \varepsilon_{N}\right) \underset{\lambda \rightarrow 0}{\longrightarrow} \mathcal{A}_{N-1}\left(\varepsilon_{2}, \ldots, \mathfrak{I}, \ldots, \varepsilon_{N}\right)
$$

with the 'soft current' $\mathfrak{I}^{\mu}$ defined as

$$
\mathfrak{I}^{\left(\lambda_{i}, \lambda_{j}\right) \mu}=-\frac{1}{\lambda} \frac{g}{\sqrt{2}}\left(\varepsilon_{j}^{\left(\lambda_{j}\right) \mu}-k_{j}^{\mu} \frac{\varepsilon_{j}^{\left(\lambda_{j}\right)} \cdot n_{a}}{k_{j} \cdot n_{a}}\right) \varepsilon_{i}^{\left(\lambda_{i}\right)} \cdot \mathfrak{j}_{\mathrm{eik}}+\mathcal{O}\left(\lambda^{0}\right),
$$

where the eikonal current is

$$
\mathrm{j}_{\mathrm{eik}}^{\mu}=\frac{k_{j}^{\mu}}{r \cdot k_{j}} .
$$

We see, that the situation is similar to the collinear limit discussed above: there is no actual factorization for the amplitude $\mathcal{A}_{N}$ since Ward identity is not satisfied due to the off-shell leg. Consequently, the piece proportional to $k_{j}^{\mu}$ in (E.30) gives a non-zero contribution. Doing, however, the same steps as for the collinear limit (notably using eq. (E.24)) we obtain

$$
\begin{aligned}
& \tilde{\mathcal{A}}_{N}\left(\varepsilon_{2}, \ldots, \varepsilon_{i}^{\left(\lambda_{i}\right)}, \varepsilon_{j}^{\left(\lambda_{j}\right)}, \ldots, \varepsilon_{N}\right) \underset{\lambda \rightarrow 0}{\longrightarrow} \\
& \tilde{\mathcal{A}}_{N-1}\left(\varepsilon_{2}, \ldots, \varepsilon_{j}^{\left(\lambda_{j}\right)}, \ldots, \varepsilon_{N}\right)\left(-\frac{1}{\lambda} \frac{g}{\sqrt{2}} \varepsilon_{i}^{\left(\lambda_{i}\right)} \cdot \mathrm{j}_{\text {eik }}\right) .
\end{aligned}
$$

Open Access. This article is distributed under the terms of the Creative Commons Attribution License which permits any use, distribution and reproduction in any medium, provided the original author(s) and source are credited. 


\section{References}

1] C. Salgado et al., Proton-Nucleus Collisions at the LHC: Scientific Opportunities and Requirements, J. Phys. G 39 (2012) 015010 [arXiv:1105.3919] [INSPIRE].

[2] FP420 R And D collaboration, M. Albrow et al., The FP420 RED Project: Higgs and New Physics with forward protons at the LHC, 2009 JINST 4 T10001 [arXiv:0806.0302] [INSPIRE].

[3] S. Catani, M. Ciafaloni and F. Hautmann, High-energy factorization and small $x$ heavy flavor production, Nucl. Phys. B 366 (1991) 135 [INSPIRE].

[4] E. Kuraev, L. Lipatov and V.S. Fadin, The Pomeranchuk Singularity in Nonabelian Gauge Theories, Sov. Phys. JETP 45 (1977) 199 [InSPIRE].

[5] I. Balitsky and L. Lipatov, The Pomeranchuk Singularity in Quantum Chromodynamics, Sov. J. Nucl. Phys. 28 (1978) 822 [inSPIRE].

[6] I. Balitsky, Operator expansion for high-energy scattering, Nucl. Phys. B 463 (1996) 99 [hep-ph/9509348] [INSPIRE].

[7] Y.V. Kovchegov, Small x F(2) structure function of a nucleus including multiple Pomeron exchanges, Phys. Rev. D 60 (1999) 034008 [hep-ph/9901281] [INSPIRE].

[8] M. Ciafaloni, Coherence Effects in Initial Jets at Small $Q^{2} / s$, Nucl. Phys. B 296 (1988) 49 [INSPIRE].

[9] S. Catani, F. Fiorani and G. Marchesini, Small x Behavior of Initial State Radiation in Perturbative QCD, Nucl. Phys. B 336 (1990) 18 [INSPIRE].

[10] K. Kutak, K. Golec-Biernat, S. Jadach and M. Skrzypek, Nonlinear equation for coherent gluon emission, JHEP 02 (2012) 117 [arXiv:1111.6928] [INSPIRE].

[11] K. Kutak, Resummation in nonlinear equation for high energy factorisable gluon density and its extension to include coherence, arXiv:1206.5757 [INSPIRE].

[12] L. Lipatov, Gauge invariant effective action for high-energy processes in QCD, Nucl. Phys. B 452 (1995) 369 [hep-ph/9502308] [INSPIRE].

[13] E. Antonov, L. Lipatov, E. Kuraev and I. Cherednikov, Feynman rules for effective Regge action, Nucl. Phys. B 721 (2005) 111 [hep-ph/0411185] [InSPIRE].

[14] G. Chachamis, M. Hentschinski, J. Madrigal Martinez and A. Sabio Vera, Quark contribution to the gluon Regge trajectory at NLO from the high energy effective action, Nucl. Phys. B 861 (2012) 133 [arXiv:1202.0649] [InSPIRE].

[15] M. Hentschinski and A.S. Vera, NLO jet vertex from Lipatov's QCD effective action, Phys. Rev. D 85 (2012) 056006 [arXiv: 1110.6741] [InSPIRE].

[16] M. Braun, L. Lipatov, M.Y. Salykin and M. Vyazovsky, Gluon production on two centers and the effective action approach, Eur. Phys. J. C 71 (2011) 1639 [arXiv:1103.3618] [INSPIRE].

[17] J. Bartels, L. Lipatov and G. Vacca, Ward Identities for Amplitudes with Reggeized Gluons, arXiv:1205.2530 [INSPIRE].

[18] M.L. Mangano, M. Moretti, F. Piccinini, R. Pittau and A.D. Polosa, ALPGEN, a generator for hard multiparton processes in hadronic collisions, JHEP 07 (2003) 001 [hep-ph/0206293] [INSPIRE]. 
[19] A. Cafarella, C.G. Papadopoulos and M. Worek, Helac-Phegas: A Generator for all parton level processes, Comput. Phys. Commun. 180 (2009) 1941 [arXiv:0710.2427] [INSPIRE].

[20] T. Gleisberg and S. Hoeche, Comix, a new matrix element generator, JHEP 12 (2008) 039 [arXiv: 0808.3674] [INSPIRE].

[21] J. Alwall, M. Herquet, F. Maltoni, O. Mattelaer and T. Stelzer, MadGraph 5: Going Beyond, JHEP 06 (2011) 128 [arXiv:1106.0522] [INSPIRE].

[22] R. Kleiss and G. van den Oord, CAMORRA: a C++ library for recursive computation of particle scattering amplitudes, Comput. Phys. Commun. 182 (2011) 435 [arXiv:1006.5614] [INSPIRE].

[23] A. Leonidov and D. Ostrovsky, Angular and momentum asymmetry in particle production at high-energies, Phys. Rev. D 62 (2000) 094009 [hep-ph/9905496] [INSPIRE].

[24] M. Deak, F. Hautmann, H. Jung and K. Kutak, Forward-Central Jet Correlations at the Large Hadron Collider, arXiv: 1012.6037 [INSPIRE].

[25] M. Deak, F. Hautmann, H. Jung and K. Kutak, Forward Jets and Energy Flow in Hadronic Collisions, Eur. Phys. J. C 72 (2012) 1982 [arXiv:1112.6354] [INSPIRE].

[26] M. Deak, F. Hautmann, H. Jung and K. Kutak, Jets in the forward region at the LHC, arXiv:0908.1870 [INSPIRE].

[27] K. Kutak, Forward Physics Hard Processes and Saturation: Theory and Phenomenology Review, Prog. Theor. Phys. Suppl. 193 (2012) 197 [arXiv:1112.4361] [INSPIRE].

[28] K. Kutak and S. Sapeta, Gluon saturation in dijet production in p-Pb collisions at Large Hadron Collider, arXiv: 1205.5035 [INSPIRE].

[29] V. Del Duca, A. Frizzo and F. Maltoni, Factorization of tree QCD amplitudes in the high-energy limit and in the collinear limit, Nucl. Phys. B 568 (2000) 211 [hep-ph/9909464] [INSPIRE].

[30] S. Catani, M. Ciafaloni and F. Hautmann, Gluon contributions to small x heavy flavor production, Phys. Lett. B 242 (1990) 97 [InSPIRE].

[31] S. Catani, M. Ciafaloni and F. Hautmann, Small x structure functions and heavy flavor production, Nucl. Phys. Proc. Suppl. 18C (1991) 220 [inSPIRE].

[32] S. Catani, M. Ciafaloni and F. Hautmann, High-energy factorization in QCD and minimal subtraction scheme, Phys. Lett. B 307 (1993) 147 [INSPIRE].

[33] R. Kleiss and W.J. Stirling, Spinor Techniques for Calculating $p \bar{p} \rightarrow W^{ \pm} / Z^{0}+$ Jets, Nucl. Phys. B 262 (1985) 235 [INSPIRE].

[34] L.J. Dixon, Calculating scattering amplitudes efficiently, hep-ph/9601359 [INSPIRE].

[35] M.L. Mangano, S.J. Parke and Z. Xu, Duality and Multi - Gluon Scattering, Nucl. Phys. B 298 (1988) 653 [inSPIRE].

[36] G. Leibbrandt, Introduction to Noncovariant Gauges, Rev. Mod. Phys. 59 (1987) 1067 [INSPIRE].

[37] F.A. Berends and W. Giele, Recursive Calculations for Processes with n Gluons, Nucl. Phys. B 306 (1988) 759 [inSPIRE].

[38] C.G. Papadopoulos and M. Worek, Multi-parton cross sections at hadron colliders, Eur. Phys. J. C 50 (2007) 843 [hep-ph/0512150] [INSPIRE]. 
[39] C. Duhr, S. Hoeche and F. Maltoni, Color-dressed recursive relations for multi-parton amplitudes, JHEP 08 (2006) 062 [hep-ph/0607057] [INSPIRE].

[40] A. Kanaki and C.G. Papadopoulos, HELAC-PHEGAS: Automatic computation of helicity amplitudes and cross-sections, hep-ph/0012004 [INSPIRE].

[41] F. Maltoni, K. Paul, T. Stelzer and S. Willenbrock, Color flow decomposition of QCD amplitudes, Phys. Rev. D 67 (2003) 014026 [hep-ph/0209271] [INSPIRE].

[42] M. Deak, F. Hautmann, H. Jung and K. Kutak, Forward Jet Production at the Large Hadron Collider, JHEP 09 (2009) 121 [arXiv:0908.0538] [INSPIRE].

[43] H. Arodz and L. Hadasz, Lectures on Classical and Quantum Theory of Fields, Springer (2010).

[44] M.L. Mangano and S.J. Parke, Multiparton amplitudes in gauge theories, Phys. Rept. 200 (1991) 301 [hep-th/0509223] [INSPIRE]. 